\title{
The $\tilde{X}^{2} B_{1},{ }^{2} B_{2},{ }^{2} A_{1}$, and ${ }^{2} A_{2}$ states of oxygen difluoride cation $\left(\mathrm{F}_{2} \mathrm{O}^{+}\right)$: High-level $a b$ initio calculations and simulation of the ultraviolet photoelectron spectrum of $\mathrm{F}_{2} \mathrm{O}$
}

\author{
De-Chao Wang, ${ }^{\text {a) }}$ Foo-Tim Chau, Daniel Kam-Wah Mok, ${ }^{\text {b) }}$ and Edmond P. F. Lee ${ }^{\text {c) }}$ \\ Department of Applied Biology and Chemical Technology, The Hong Kong Polytechnic University, \\ Hung Hom, Kowloon, Hong Kong \\ Levi Beeching, J. Steven Ogden, and John M. Dyke ${ }^{\mathrm{b}), \mathrm{d})}$ \\ Department of Chemistry, University of Southampton, Highfield, Southampton SO17, 1BJ, United Kingdom
}

(Received 17 January 2001; accepted 3 April 2001)

\begin{abstract}
The ultraviolet photoelectron spectrum of $\mathrm{F}_{2} \mathrm{O}$ was recorded with a higher resolution than previously published. New vibrational structure was observed in the second and third bands. Near state-of-the-art molecular orbital calculations were performed on the $\tilde{X}^{1} A_{1}$ state of $\mathrm{F}_{2} \mathrm{O}$ and the $\tilde{X}^{2} B_{1},{ }^{2} B_{2},{ }^{2} A_{1}$, and ${ }^{2} A_{2}$ state of $\mathrm{F}_{2} \mathrm{O}^{+}$, and their potential energy functions were computed. Spectral simulations based on Franck-Condon factor calculations including the Duchinsky effect were carried out within the harmonic oscillator model and also with the inclusion of anharmonicity, in order to assist spectral assignment. Based on the computed ionization energies obtained with the coupled cluster and multireference configuration interaction methods with basis sets of up to quintuple zeta quality, the order of the low-lying cationic states of $\mathrm{F}_{2} \mathrm{O}^{+}$has been firmly established. However, the detailed assignment of the overlapping second and third photoelectron bands was only achieved with the aid of spectral simulation. The iterative Franck-Condon analysis (IFCA) procedure was carried out for the first band $\left\{\mathrm{F}_{2} \mathrm{O}^{+}\left(\tilde{X}^{2} B_{1}\right) \leftarrow \mathrm{F}_{2} \mathrm{O}\left(\tilde{X}^{1} A_{1}\right)\right\}$ in the photoelectron spectrum. With the geometrical parameters of $\mathrm{F}_{2} \mathrm{O}\left(\tilde{X}^{1} A_{1}\right)$ being fixed at the available experimental values, geometrical parameters of the $\widetilde{X}^{2} B_{1}$ state of $\mathrm{F}_{2} \mathrm{O}^{+}$were derived. Based on anharmonic Franck-Condon factor calculations, the recommended IFCA geometrical parameters for the ground state of $\mathrm{F}_{2} \mathrm{O}^{+}$are $R(\mathrm{FO})=1.323 \pm 0.002 \AA$ and $\angle \mathrm{FOF}=107.3 \pm 0.2^{\circ}$. (C) 2001 American Institute of Physics. [DOI: 10.1063/1.1373690]
\end{abstract}

\section{INTRODUCTION}

The He I photoelectron (PE) spectrum of $\mathrm{F}_{2} \mathrm{O}$ was reported by Cornford and co-workers in 1971. ${ }^{1}$ The observed bands were assigned with the aid of results of INDO and $\mathrm{CNDO} / 2$ semi-empirical calculations, and previous $a b$ initio calculations (see Ref. 1 for details). A year later, Brundle and co-workers ${ }^{2}$ reported a very similar He I PE spectrum of $\mathrm{F}_{2} \mathrm{O}$, perhaps with a slightly better resolution. Ab initio calculations were performed, and both the Koopmans' Theorem (KT) and the $\Delta$ self-consistent field (SCF) method were utilized to assign the observed spectrum. The assignments of the first four PE bands from these two studies are essentially identical, giving the order of the lowest four states of $\mathrm{F}_{2} \mathrm{O}^{+}$ as ${ }^{2} B_{1},{ }^{2} A_{1},{ }^{2} B_{2}$, and ${ }^{2} A_{2}$. These cationic states were assigned to four distinct PE bands observed in the He I PE spectra, with measured vertical ionization energies (VIEs) of $13.25,16.10,16.44$, and $18.50 \mathrm{eV}$, respectively (from Ref. 2; see also Table I). However, Brundle and co-workers ${ }^{2}$ also suggested an alternative assignment. Since the ionization

\footnotetext{
a) Also at: Center of Analysis and Test, Sichuan Normal University, Chengdu, Sichuan, People's Republic of China.

b) Author to whom correspondence should be addressed.

c)Also at: Department of Chemistry, University of Southampton, Highfield, Southampton SO17, 1BJ, United Kingdom.

${ }^{\mathrm{d})}$ Electronic mail: jmdyke@ soton.ac.uk
}

leading to the ${ }^{2} A_{2}$ state corresponds to an electron being removed from the nonbonding $F$-localized $a_{2}$ molecular orbital, this band is expected to be sharp. In view of this consideration, the ${ }^{2} A_{2}$ ionic state might be more appropriately assigned to the relatively sharp third PE band at $16.44 \mathrm{eV}$, rather than the broad and relatively weak band at a significantly higher VIE of $18.50 \mathrm{eV}$. Thus, the ${ }^{2} A_{1},{ }^{2} B_{2}$, and ${ }^{2} A_{2}$ states of $\mathrm{F}_{2} \mathrm{O}^{+}$would be assigned to the observed overlapping second and third PE bands with measured VIEs of 16.10 and $16.44 \mathrm{eV}$, respectively. With this alternative assignment, the precise positions of the lowest ${ }^{2} A_{1},{ }^{2} B_{2}$, and ${ }^{2} A_{2}$ states of $\mathrm{F}_{2} \mathrm{O}^{+}$are unclear, as three states are now assigned to two observed bands with no resolved vibrational structure.

Since the publication of these two He I PE studies on $\mathrm{F}_{2} \mathrm{O},{ }^{1,2}$ numerous theoretical studies, which calculated the ionization energies (IEs) of the low-lying states of $\mathrm{F}_{2} \mathrm{O}^{+}$by various methods, have appeared in the literature. Some of the computed VIEs reported for the lowest four cationic states ${ }^{1-9}$ are summarized in Table I (see footnote a). From Table I, it is clear that the assignment of the ${ }^{2} B_{1}$ state of $\mathrm{F}_{2} \mathrm{O}^{+}$to the first observed $\mathrm{PE}$ band is reasonably certain. However, based on available calculations, the order of the next three cationic states is far from unambiguous. Regarding the alternative assignment of the position of the ${ }^{2} A_{2}$ state mentioned by 
TABLE I. The vertical ionization energies (VIEs in eV) to the first four cationic states of $\mathrm{F}_{2} \mathrm{O}^{+}$from $\mathrm{F}_{2} \mathrm{O}\left(X^{1} A_{1}\right)$ from previous calculations.

\begin{tabular}{|c|c|c|c|c|c|c|}
\hline Method $^{\mathrm{a}}$ & ${ }^{2} B_{1}$ & ${ }^{2} B_{2}$ & ${ }^{2} A_{1}$ & ${ }^{2} A_{2}$ & Ref. & First author/Year \\
\hline $\mathrm{INDO}^{\mathrm{b}}$ & 14.3 & 16.7 & 15.8 & 18.1 & 1 & Cornford/1971 \\
\hline$\Delta \mathrm{SCF} / \mathrm{DZ} \mathrm{C}^{\mathrm{c}}$ & 13.5 & 16.9 & 16.3 & 18.3 & 2 & Brundle/1972 \\
\hline MRDCI/DZP $+b^{d}$ & 13.43 & 16.17 & 16.36 & 17.41 & 3 & Valenta/1980 \\
\hline $\mathrm{ROCI}+\mathrm{Q}^{\mathrm{e}} / \mathrm{DZP}$ & 13.18 & 16.01 & 16.59 & 16.62 & 4 & Langhoff/1981 \\
\hline RSPT-KT $/ \mathrm{DZ}-\mathrm{STO}$ & 13.29 & 16.54 & 16.33 & 16.69 & 5 & Chong/1974 \\
\hline $\mathrm{GF}^{\mathrm{g}}$ & 13.06 & 16.46 & 16.37 & 16.95 & 6 & Von Niessen/1979 \\
\hline RSPT-KT ${ }^{\mathrm{h}} / \mathrm{DZP}$ & 13.14 & 16.78 & 16.75 & $17.14^{\mathrm{i}}$ & 7 & Langhoff/1982 \\
\hline LDA-rTS $/$ ANO & 13.30 & 16.10 & 16.48 & 16.23 & 8 & Duffy/1993 \\
\hline $\mathrm{pGW} 2^{\mathrm{k}}$ & 13.39 & 16.27 & 16.61 & 16.54 & 9 & $\mathrm{Hu} / 1997$ \\
\hline Observed $^{\mathrm{m}}$ & 13.26 & 16.47 & 16.17 & 18.68 & 1 & Cornford/1971 \\
\hline Observed $^{1}$ & 13.25 & 16.44 & 16.10 & 18.50 & 2 & Brundle/1972 \\
\hline
\end{tabular}

${ }^{\mathrm{a}}$ The highest level of calculation from the respective reference.

${ }^{\mathrm{b}}$ For earlier calculations, see Ref. 1 and references therein.

${ }^{\mathrm{c}}$ Whether the irreducible representation $b_{1}$ or $b_{2}$ in the $C_{2 V}$ point group corresponds to being symmetrical with respect to the molecular plane, depends on the choice of the axes system. Brundle and co-workers (Ref. 2) employed an axes system, which differs from others. Here, the convention that $b_{2}$ corresponds to being symmetrical with respect to the molecular plane is used.

${ }^{d}$ sp bond functions (exponent $=1.0$ ) at the center of the mass of the two OF bonds were included in the basis set.

${ }^{\mathrm{e}} \mathrm{RO}$ refers to relaxed orbitals, which mean that the molecular orbitals of the ground state of the cation (instead of those of the neutral molecule) were employed as the many particle basis set in the CI calculation. $Q$ refers to the Davidson correction for quadruple excitations.

${ }^{\mathrm{f}}$ Third order Rayleigh-Schrodinger perturbation corrections to Koopman's Theorem (KT). STO denotes Slater-type orbitals.

${ }^{\mathrm{g}}$ Green function calculation.

${ }^{\mathrm{h}}$ As footnote $\mathrm{f} ; \Delta\left(E^{\mathrm{GA}}\right)$ values (see original work for details).

${ }^{\mathrm{i}} \mathrm{A}$ best estimated VIE for the ${ }^{2} A_{2}$ state was given to be $16.6 \mathrm{eV}$.

${ }^{j}$ Restricted transition state method employing the deMon density functional program; an observed VIE value of $16.32 \mathrm{eV}$ was given to the ${ }^{2} B_{2}$ state in this work, which was quoted from the original work of Cornford and co-workers (Ref. 1), but we are unable to trace the origin of this value.

${ }^{k}$ Parametrized second order Green function (pG) times screened interaction (W2) approximation (see original work for details). It is noted that in this work, the assignments given for the observed VIEs from Cornford and co-workers (Ref. 1) are not the same as those in the original paper. Some typing errors in Table II of this article of $\mathrm{Hu}$ and co-workers (Ref. 9) are suspected. See also footnote j for the observed VIE value of the ${ }^{2} B_{2}$ state given in this work. ${ }^{1}$ Assignments based on KT from previous $a b$ initio and their own semi-empirical calculations.

${ }^{\mathrm{m}}$ Assignments based on $\mathrm{KT}$ and $\Delta \mathrm{SCF}$ results; an alternative assignment of the ${ }^{2} A_{2}$ state to the relatively sharp peak at $16.44 \mathrm{eV}$ was given, because of the nonbonding character of the $1 a_{2}$ molecular orbital; see text.

Brundle and co-workers, ${ }^{2}$ some calculated VIEs (e.g., those obtained by the ROCI $+\mathrm{Q}$, LDA-rTS and pGW2 methods; see Table I) clearly support it, but for the others, there is no clear preference between the original ${ }^{1,2}$ and the alternative assignments. ${ }^{2}$ The computed value obtained from the highest level of calculation prior to the present study is that obtained by Valenta and co-workers ${ }^{3}$ from a MRDCI study. Their VIE value of $17.41 \mathrm{eV}$ for ionization to the ${ }^{2} A_{2}$ state can be considered to favor the original assignment of associating the ${ }^{2} A_{2}$ ionic state to the broad band at $18.50 \mathrm{eV}$.

In view of these considerations, it is the purpose of the present investigation to provide more reliable assignments for the observed PE spectra of $\mathrm{F}_{2} \mathrm{O}$ and to obtain more precise positions of the low-lying states of $\mathrm{F}_{2} \mathrm{O}^{+}$by performing high-level $a b$ initio calculations and carrying out spectral simulations. For the ${ }^{2} A_{2}$ state, the two proposed assignments would have their respective experimental VIEs differing by over $2 \mathrm{eV}$. It is expected that current near state-of-the-art quantum chemical calculations should give IEs, with uncertainties of less than $0.2 \mathrm{eV}$, and hence should be able to determine which of the two proposed assignments of the ${ }^{2} \mathrm{~A}_{2}$ state is more reliable. In addition, we propose to record the $\mathrm{He}$ I PE spectrum of $\mathrm{F}_{2} \mathrm{O}$ again with the intention of obtaining a better resolved spectrum than previously reported, in order to resolve vibrational structure, which was unresolved previously in the second and third bands. With a higher quality experimental spectrum and the assistance of spectral simulations, it is hoped that the assignments and the posi- tions of the four lowest-lying cationic states of $\mathrm{F}_{2} \mathrm{O}^{+}$could be firmly established in the present study.

Recently, we have reported related investigations on the He I PE spectra of $\mathrm{Cl}_{2} \mathrm{O}$ (Ref. 10) and $\mathrm{ClO}_{2}$ (Ref. 11) by $a b$ initio calculations and spectral simulation. For $\mathrm{Cl}_{2} \mathrm{O}$, which is valence isoelectronic with $\mathrm{F}_{2} \mathrm{O}$, the first four observed $\mathrm{PE}$ bands are well resolved with vibrational structure, ${ }^{12}$ and their assignments have been firmly established to be due to the lowest ${ }^{2} B_{1},{ }^{2} B_{2},{ }^{2} A_{1}$, and ${ }^{2} A_{2}$ cationic states respectively. Comparing the assignments of the observed bands in the PE spectra of $\mathrm{Cl}_{2} \mathrm{O}$ with those of $\mathrm{F}_{2} \mathrm{O}$ (original assignments from Refs. 1 and 2), the following points should be noted. First, the order of the second and third cationic states is reversed. Second, the fourth PE band, assigned to ionization to the ${ }^{2} A_{2}$ state of $\mathrm{Cl}_{2} \mathrm{O}^{+}$has a very strong $0,0,0-0,0,0$ vibrational component, while the third PE band assigned to ionization to the ${ }^{2} A_{1}$ state is broad and relatively weak. In addition, the third $\left({ }^{2} A_{1}\right)$ and fourth $\left({ }^{2} A_{2}\right)$ bands are close to, and overlapping with, each other.

For $\mathrm{ClO}_{2}$, the second $\mathrm{PE}$ band is a heavily overlapped band, ${ }^{13}$ with contributions from ionization to five cationic states. Nevertheless, with the aid of spectral simulations, which employ our recently developed anharmonic FranckCondon (FC) code, ${ }^{11}$ most of the observed vibrational structure of this band could be adequately accounted for, and a reasonably reliable order of the five cationic states involved was proposed. In the present study on the He I PE spectrum 
of $\mathrm{F}_{2} \mathrm{O}$, an approach similar to the aforementioned two investigations ${ }^{10,11}$ has been employed.

The equilibrium structure of neutral $\mathrm{F}_{2} \mathrm{O}$ has been determined by microwave spectroscopy; ${ }^{14,15}$ the OF bond distance is $1.4053 \AA$, and the bond angle is $103.07^{\circ}$. The harmonic and anharmonic force fields of $\mathrm{F}_{2} \mathrm{O}$ have received considerable attention. ${ }^{16-20}$ Since $\mathrm{F}_{2} \mathrm{O}$ is in the test set of molecules, whose heats of formation were calculated by the composite method, $G 2,{ }^{21}$ many calibration studies ${ }^{22,23}$ on alternative theoretical methods, such as RI-MP $2,{ }^{24} G 3,{ }^{25}$ and some density functional methods with new functionals, ${ }^{26-32}$ have included $\mathrm{F}_{2} \mathrm{O}$. However, the cation, $\mathrm{F}_{2} \mathrm{O}^{+}$, has received significantly less attention.

\section{THEORETICAL CONSIDERATIONS AND COMPUTATIONAL DETAILS}

\section{A. $\mathbf{A b}$ initio calculations}

Initially, geometry optimization and harmonic vibrational frequency calculations were carried out on the ground state of neutral $\mathrm{F}_{2} \mathrm{O}$ and the four lowest-lying states of $\mathrm{F}_{2} \mathrm{O}^{+}$. The complete active space self-consistent field (CASSCF), B3LYP, MP2, and QCISD methods were used with various standard basis sets. The GAUSSIAN98 suite of programs ${ }^{33}$ was employed for these calculations. In order to obtain more reliable geometries, RCCSD(T)/cc-pVTZ and/or RCCSD(T)/ cc-pVQZ geometry optimization calculations were carried out, using the MOLPRO ${ }^{34,35}$ suite of programs. For relative energies, adiabatic ionization energies (AIEs) and/or VIEs were evaluated up to the RCCSD(T)/cc-pV5Z//RCCSD(T)/ cc-pVQZ level and also were obtained by various extrapolation [to the complete basis set (CBS) limit] techniques. ${ }^{36-39}$ In addition, CASSCF/MRCI/cc-pVQZ//RCCSD(T)/ccpVQZ calculations were also carried out using MOLPRO ${ }^{35,40}$ to assess the effect of the multireference character on the computed IEs. The optimized geometrical parameters, computed force constants and AIEs obtained at their respective highest levels of calculation were then employed in the subsequent Franck-Condon factor (FCF) calculations, which are based on the harmonic oscillator model and include the Duschinsky effect (for details, see Refs. 41 and 42).

For reasons, which will be discussed in Sec. IV, the anharmonic FC code was also employed for spectral simulation. ${ }^{11}$ The asymmetric stretching mode, $v_{3}$, has not been considered in the anharmonic FCF calculations, because harmonic FCF calculations gave negligible intensities for excitation of this vibrational mode upon ionization to all four lowest cationic states. For the ground state neutral and the four lowest ionic states, energy scans are performed to obtain the potential energy functions (PEFs), equilibrium geometrical parameters, and fundamental frequencies of the symmetric stretch and bending modes (see Sec. II B).

\section{B. Determination of potential energy surfaces}

The PEFs employed in the vibrational Schrodinger equation for the variational calculation of the vibrational wave functions (see Sec. II C) are determined by fitting the follow- ing polynomial to different numbers of $\mathrm{RCCSD}(\mathrm{T})$ single energy points (depending on the electronic states involved; as will be discussed later),

$$
V=\sum_{i j} C_{i j}\left(S_{1}\right)^{i}\left(S_{2}\right)^{j}+V_{\text {eqm }} \cdot
$$

The PEFs are expressed in the displacements of symmetry coordinates

$$
S_{1}=\left(\Delta r_{1}+\Delta r_{2}\right) / \sqrt{2},
$$

and the bending coordinates suggested by Carter and Handy, ${ }^{43}$

$$
S_{2}=\Delta \theta+\alpha \Delta \theta^{2}+\beta \Delta \theta^{3},
$$

where $\Delta r_{1,2}$ and $\Delta \theta$ are displacements in $\mathrm{FO}$ bond lengths (for the symmetric stretch, $\Delta r_{1}=\Delta r_{2}$ ) and FOF bond angle, respectively. By restricting the energy gradient in $S_{2}$ to zero when the molecule is linear (i.e., when $\theta=\pi$ ), an expression relating $\alpha$ and $\beta$ can be obtained:

$$
\beta=\left[1+3 \alpha\left(\pi-\theta_{\text {eqm }}\right)^{2}\right] /\left[-2\left(\pi-\theta_{\text {eqm }}\right)\right] .
$$

This condition is imposed to make sure that the gradient of the resulting potential energy function takes a value of zero when the molecule is linear. The nonlinear least square fit procedure, NL2SOL (Ref. 44) was employed to obtain the $C_{i j}$ 's, $V_{\text {eqm }}, r_{\text {eqm, }} \theta_{\text {eqm }}$, and $\alpha$ values from the computed single point energies.

In all the PEFs, all terms up to the fourth order and also $C_{05}$ and $C_{06}$ were included. For the ground states of $\mathrm{F}_{2} \mathrm{O}$ and $\mathrm{F}_{2} \mathrm{O}^{+}$, as the vibrational structure observed in the He I PE spectrum is essentially due to the symmetric stretching mode, the fifth and sixth order terms for stretching, $C_{50}$ and $C_{60}$, were included as well. The numbers of single point energies evaluated were 26 for the ground states of $\mathrm{F}_{2} \mathrm{O}$ and $\mathrm{F}_{2} \mathrm{O}^{+}, 29$ for the ${ }^{2} B_{2}$ ionic state and 24 for the ${ }^{2} A_{1}$ and ${ }^{2} A_{2}$ ionic states. From the harmonic spectral simulation, the bending mode progression of the ${ }^{2} B_{2}$ state is rather long, so more points are used along the bending mode of the ${ }^{2} B_{2}$ state. A higher level of theory, RCCSD(T)/aug-cc-pVQZ, was employed to determine the PEFs of the neutral and cationic ground states, so that a more reliable cationic geometry can be deduced using the iterative Franck-Condon analysis (IFCA) procedure (see Sec. IIC). The energy scans of the neutral and cationic ground states covered the range, $-0.21 \AA \leqslant \Delta r \leqslant+0.35 \AA$ and $30^{\circ} \leqslant \Delta \theta \leqslant+30^{\circ}$. For the ${ }^{2} B_{2},{ }^{2} A_{1}$, and ${ }^{2} A_{2}$ cationic states, the RCCSD(T)/aug-ccpVTZ level of calculation was used. The energy scans of these cationic states covered the range, $-0.14 \AA \leqslant \Delta r \leqslant$ $+0.21 \AA$ and $30^{\circ} \leqslant \Delta \theta \leqslant+30^{\circ}$.

\section{Anharmonic FC code (AN-FCF) and spectral simulation}

The variational calculation of the anharmonic vibrational wavefunction and the AN-FCF method have been reported previously (see Ref. 11 for details). Only a brief description and some technical details specific to the present study are given here. 


\section{Variational calculation of anharmonic vibrational wave functions}

The anharmonic vibrational wave function of the $m$ th vibrational state is expressed as follows,

$$
|\mathbf{m}\rangle=\sum c_{\mathbf{m}, v} \phi\left(v_{1}\right) \phi\left(v_{2}\right) \phi\left(v_{3}\right),
$$

where $c_{\mathbf{m}, v}$ are the expansion coefficients, the subscript $v$ denotes $\left(v_{1}, v_{2}\right.$, and $\left.v_{3}\right)$ and $\phi\left(v_{i}\right)$ are the $v$ th order harmonic oscillator functions of the normal mode $i$. The expansion coefficients, $c_{\mathbf{m}, v}$, are obtained by diagonalizing Watson's Hamiltonian. ${ }^{45,46}$ The anharmonicities of the vibrational motions are included in the present formalism via the anharmonic PEFs and the anharmonic vibrational wave functions defined in Eq. (2). The Hamiltonian matrix elements, $\langle n|H| m\rangle$, are evaluated using the Gauss-Hermite quadrature along each normal mode. Since the ab initio PEFs just described are not expressed in normal coordinates, each quadrature grid point is transformed to the coordinate system of the PEF.

For neutral $\mathrm{F}_{2} \mathrm{O}$, vibrational levels with quantum numbers up to 10 were considered in the variational calculations, with a constraint of $v_{1}+v_{2} \leqslant 10$. This was shown to be more than sufficient from a series of calculations including different numbers of vibrational levels. Similarly, for calculations involving the $\mathrm{F}_{2} \mathrm{O}^{+}$electronic states, vibrational levels with quantum number of up to 25 were included, with a constraint of $v_{1}+v_{2} \leqslant 25$. Thus, a total of 66 harmonic basis functions for the neutral $\mathrm{F}_{2} \mathrm{O}\left(\tilde{X}^{1} A_{1}\right)$ and of 351 harmonic basis functions for the cationic states have been used in the variational calculations to obtain the anharmonic vibrational wave functions as given by Eq. (2). FCF calculations (see Sec. II C 2) employing vibrational wave functions obtained with larger harmonic basis sets for the ground neutral state and ground cationic state were also carried out to test the sensitivity of calculated FCFs and simulated PE bands to the selected basis sets. These calculations show that larger basis sets than the ones just mentioned do not alter the quality of the calculated FCFs or the spectral simulation to any significant extent.

\section{Anharmonic FCF calculation, IFCA procedure and spectral simulation}

The anharmonic FCF is expressed as

$$
\begin{aligned}
\operatorname{FCF}(\mathbf{m}, \mathbf{n}) & =\langle\mathbf{m} \mid \mathbf{n}\rangle^{2} \\
& =\left(\sum_{\mathbf{v}^{\prime}, \mathbf{v}^{\prime \prime}} c_{\mathbf{m}, \mathbf{v}^{\prime}} c_{\mathbf{n}, \mathbf{v}^{\prime \prime}}\left\langle v_{1}^{\prime}, v_{2}^{\prime}, v_{3}^{\prime} \mid v_{1}^{\prime \prime}, v_{2}^{\prime \prime}, v_{3}^{\prime \prime}\right\rangle\right)^{2},
\end{aligned}
$$

where the primed and double primed quantities correspond to those of the final state and the initial state, respectively. The expansion coefficients $c_{\mathbf{m}, \mathbf{v}^{\prime}}$ and $c_{\mathbf{n}, \mathbf{v}^{\prime \prime}}$ are determined by the variational calculation mentioned, and $\left\langle v_{1}^{\prime}, v_{2}^{\prime}, v_{3}^{\prime} \mid v_{1}^{\prime \prime}, v_{2}^{\prime \prime}, v_{3}^{\prime \prime}\right\rangle$ is the overlap integral of the corresponding harmonic functions. Since the anharmonic wave functions are expressed as linear combinations of harmonic functions in normal coordinates, the overlap integral can be evaluated readily using the method of Chen. ${ }^{41}$ Since the
Duschinsky rotation matrix is included in this method, the anharmonic FCF obtained in this way has incorporated the effect of anharmonicity and Duschinsky rotation.

With the equilibrium geometry of the ground state of $\mathrm{F}_{2} \mathrm{O}$ available experimentally, ${ }^{14,15}$ the IFCA procedure can be applied to derive the geometry of the cationic states, as in previous studies. ${ }^{10,11,47,48}$ In the IFCA procedure, the geometry of the neutral molecule is fixed at the available experimental geometry, while the cationic state geometrical parameters are adjusted systematically (initially from the predicted $a b$ initio geometry changes), until the simulated spectrum matches the experimental one. The IFCA procedure can be carried out with both the harmonic and AN-FCF method. In the latter case, during the IFCA procedure, the shape of the ab initio PEFs of the two electronic states involved are kept unchanged (for further details, see Ref. 11). The IFCA procedure was applied to the first PE band, where well resolved vibrational structure was observed. Experimentally derived geometrical parameters of the $\widetilde{X}^{2} B_{1}$ state were thus obtained (see Sec. III). For the second and third bands, the observed vibrational structure is poorly resolved and the AIE positions of the three cationic states involved are uncertain from the observed spectrum. Consequently, the IFCA procedure was only applied to the ionization leading to the ${ }^{2} A_{2}$ state (the fourth ionic state) in order to obtain a qualitative match between the observed and simulated spectra (see Sec. III).

Gaussian functions with the appropriate full width half maximum (FWHM), and relative intensities as given by the computed FCFs were used to simulate the vibronic peaks in the He I PE spectrum. For the bands corresponding to ionization to the ${ }^{2} B_{2},{ }^{2} A_{1}$, and ${ }^{2} A_{2}$ ionic states, their individual simulated band areas were assumed to be the same. This was felt to be a reasonable approximation as the molecular orbitals associated with these ionizations are composed of $O 2 p$ and $F 2 p$ atomic orbitals which are expected to have very similar cross sections at the He I photon energy. For the first $\mathrm{PE}$ band of $\mathrm{F}_{2} \mathrm{O}$, the experimental AIE was used in the simulation. For the three excited cationic states considered in this work, the RCCSD(T)/cc-pV5Z//RCCSD(T)/cc-pVQZ AIEs were employed initially. Subsequently, some small adjustments in band positions were made in order to obtain a reasonable match between the observed and simulated spectra. The details of these adjustments are discussed in the Sec. IV.

\section{EXPERIMENT}

\section{A. Synthesis of $F_{2} O$}

The preparation of oxygen difluoride was based on the original procedures described by Ruff and Menzel, ${ }^{49}$ and Lebeau and Damiens. ${ }^{50}$ In a typical synthesis, helium gas containing $5 \% \mathrm{~F}_{2}$ was bubbled through a solution of $0.5 \mathrm{M}$ $\mathrm{NaOH}$ at atmospheric pressure. The resultant gases were then allowed to pass firstly through a U-tube at ca. $200 \mathrm{~K}$ to remove $\mathrm{H}_{2} \mathrm{O}$, and then through a spiral trap at $77 \mathrm{~K}$, before finally emerging via a bubbler containing aqueous KI solution. Pyrex vessels and tubing fitted with polytetrafluoroethylene taps and joints proved to be quite satisfactory at the low partial pressures of $\mathrm{F}_{2}$ and $\mathrm{F}_{2} \mathrm{O}$ used in the system. 
TABLE II. The minimum-energy geometrical parameters (bond length in $\AA$ /bond angle in degrees) for the neutral $\left(\tilde{X}^{1} A_{1}\right)$ and the four lowest-lying cationic states $\left(\tilde{X}^{2} B_{1},{ }^{2} B_{2},{ }^{2} A_{1},{ }^{2} A_{2}\right)$ of $\mathrm{F}_{2} \mathrm{O}$.

\begin{tabular}{|c|c|c|c|c|c|}
\hline Methods & $\tilde{X}^{1} A_{1}$ & $\tilde{X}^{2} B_{1}$ & ${ }^{2} B_{2}$ & ${ }^{2} A_{1}$ & ${ }^{2} \mathrm{~A}_{2}$ \\
\hline MP2/cc-pVTZ & $1.398 / 103.15$ & $1.2715 / 107.99$ & $1.3714 / 79.92$ & $1.3177 / 121.25$ & $1.5096 / 104.93$ \\
\hline B3LYP/cc-pVTZ & $1.4036 / 103.97$ & 1.3186/108.01 & $1.4280 / 84.96$ & $1.3777 / 118.71$ & $1.4462 / 98.48$ \\
\hline QCISD/cc-pVTZ & $1.3945 / 103.15$ & $1.3178 / 107.29$ & $1.4200 / 83.48$ & $1.3794 / 119.17$ & $\mathrm{a}$ \\
\hline $\operatorname{CCSD}(\mathrm{T}) / \mathrm{cc}-\mathrm{pVTZ}$ & $1.4056 / 103.04$ & $1.3238 / 107.43$ & $1.4416 / 82.71$ & $1.3360 / 118.89$ & $1.4681 / 98.54$ \\
\hline $\operatorname{CCSD}(\mathrm{T}) / \mathrm{cc}-\mathrm{pVQZ}$ & & & $1.4383 / 82.46$ & $1.3613 / 118.88$ & $1.4644 / 98.20$ \\
\hline $\operatorname{CCSD}(\mathrm{T}) /$ aug-cc-pVTZ & & & $1.4437 / 82.28$ & $1.3689 / 118.57$ & $1.4698 / 97.94$ \\
\hline $\operatorname{CCSD}(\mathrm{T}) /$ aug-cc-pVQZ & $1.4056 / 103.04$ & $1.3190 / 107.28$ & & & \\
\hline $\begin{array}{l}\operatorname{CASSCF}(7,6) / 6- \\
311+\mathrm{G}(2 \mathrm{~d})\end{array}$ & & & & & $1.5098 / 94.70$ \\
\hline $\operatorname{CASSCF}(7,6) / c c-p V T Z$ & & & & & $1.5023 / 95.08$ \\
\hline $\operatorname{CCSD}(\mathrm{T}) / \mathrm{cc}-\mathrm{pVTZ} \mathrm{Z}^{\mathrm{c}}$ & $1.4056 / 103.09$ & & & & \\
\hline $\operatorname{CCSD}(\mathrm{T}) / \mathrm{cc}-\mathrm{pVQZ}{ }^{\mathrm{c}}$ & $1.4036 / 103.10$ & & & & \\
\hline IFCA $^{\mathrm{d}}$ & & $1.331 / 107.3$ & & & \\
\hline IFCA $^{\mathrm{e}}$ & & $1.323 / 107.3$ & & & $1.460 / 95.0$ \\
\hline Experimental values $^{\mathrm{f}}$ & $1.4053 / 103.07$ & & & & \\
\hline
\end{tabular}

${ }^{\mathrm{a}} \mathrm{QCISD}$ excessive iterations in the numerical second derivative calculations.

${ }^{\mathrm{b}}$ The optimized geometrical parameters were from energy scans and PEF fittings.

${ }^{\mathrm{c}}$ Reference 19.

${ }^{\mathrm{d}}$ The IFCA geometry from the harmonic IFCA procedure (see text for details).

${ }^{\mathrm{e}}$ The IFCA geometry from the anharmonic IFCA procedure (see text for details); these are the recommended values in this work

${ }^{\mathrm{f}}$ References 14 and 15 ; these are equilibrium values.

The $\mathrm{He} / \mathrm{F}_{2}$ flow rate was held at ca. $20 \mathrm{ml} / \mathrm{min}$, and after ca. 3-4 hr, small quantities of liquid $\mathrm{F}_{2} \mathrm{O}$ could be observed in the spiral trap. At this temperature $(77 \mathrm{~K}), \mathrm{F}_{2} \mathrm{O}$ has a finite vapor pressure (ca. $1 \mathrm{mbar}$ ), and also has the capacity to dissolve $\mathrm{O}_{2}$, which is formed as a byproduct in the $\mathrm{F}_{2} / \mathrm{NaOH}$ reaction. The final purification step, which involves the removal of dissolved $\mathrm{O}_{2}$ under reduced pressure, therefore results in significant loss of $\mathrm{F}_{2} \mathrm{O}$. However, after this purification step, $\mathrm{F}_{2} \mathrm{O}$ was the only volatile material remaining at 77 $\mathrm{K}$, and it was therefore admitted to the spectrometer inlet system directly from the $77 \mathrm{~K}$ trap. This procedure resulted in He I PE spectra, which effectively showed no impurities other than the normal background trace amounts of $\mathrm{N}_{2}$. Sample purity was also checked by matrix isolation infrared spectroscopy, and no other products were detected.

\section{B. He I PE spectrometer}

The He I PE spectrometer used has been described previously. ${ }^{51}$ Briefly, it consists of a $150^{\circ}$ sector hemispherical analyzer, a differentially pumped ionization chamber, a capillary He discharge light source and a channeltron detector. The operational resolution is better than $30 \mathrm{meV}$ FWHM for Ar. Low partial pressures of $\mathrm{O}_{2}$ and $\mathrm{N}_{2}$ were introduced into the ionization chamber as internal calibrants for the first, and the second and third bands of $\mathrm{F}_{2} \mathrm{O}$, respectively.

\section{RESULTS AND DISCUSSION}

\section{A. Ab initio calculations: Minimum-energy geometry and vibrational frequencies}

The minimum-energy geometries, computed harmonic vibrational frequencies, and IEs obtained in this work for $\mathrm{F}_{2} \mathrm{O}\left(\tilde{X}^{1} A_{1}\right)$ and the low-lying states of $\mathrm{F}_{2} \mathrm{O}^{+}$are summarized in Tables II-IV, respectively, and compared with ex- perimental values where available. From Table II, it can be seen that the calculated geometrical parameters of neutral $\mathrm{F}_{2} \mathrm{O}\left(\tilde{X}^{1} A_{1}\right)$ readily converge to the available experimental values as the level of calculation improves. At the highest level, CCSD(T)/aug-cc-pVQZ, the calculated geometrical parameters are very close to the experimental values.

For the cationic states considered here, no experimentally derived geometries were available prior to the present study. From Table II, it seems clear that, for all the cationic states considered, the optimized geometrical parameters are rather sensitive to the levels of calculations used in obtaining them. This is particularly true for the ${ }^{2} A_{2}$ state and this will be further discussed later. A higher level of calculation is clearly required to obtain reliable geometrical parameters for the cationic states than the neutral ground state. A similar conclusion can be drawn from Table III for the computed vibrational frequencies. In particular, the MP2 level is inadequate for all the cationic states studied, giving both geometrical parameters and harmonic vibrational frequencies significantly different from those obtained from higher levels of calculation. In contrast, the B3LYP results are in closer agreement with the QCISD and/or RCCSD(T) results. For the ${ }^{2} A_{2}$ ionic state, CASSCF calculations were carried out to obtain the minimum-energy geometry and harmonic vibrational frequencies, because QCISD geometry optimization on this state faced excessive QCISD iteration problems; in addition, both MP2 and B3LYP frequency calculations yielded an imaginary frequency for the $b_{2}$ asymmetric stretching mode. The CASSCF calculations on the ${ }^{2} A_{2}$ state considered seven electrons in six active orbitals. The second configuration interaction CI root was requested in order to obtain the ${ }^{2} A_{2}$ ionic state (a similar approach was employed in our study on the ${ }^{2} A_{2}$ state of $\mathrm{Cl}_{2} \mathrm{O}^{+}$; see Ref. 10). With both the $6-311+\mathrm{G}(2 d)$ and cc-pVTZ basis sets, the 
TABLE III. The computed harmonic vibrational frequencies $\left[\omega_{1}\left(a_{1}\right.\right.$ symmetric stretch $) / \omega_{2}\left(a_{1}\right.$ symmetric bending $) / \omega_{3}\left(b_{2}\right.$ asymmetric stretch $)$ in $\left.\mathrm{cm}^{-1}\right]$ for the neutral $\left(\tilde{X}^{1} A_{1}\right)$ and the four lowest energy cationic states $\left(\tilde{X}^{2} B_{1},{ }^{2} B_{2},{ }^{2} A_{1}\right.$, and $\left.{ }^{2} A_{2}\right)$ of $\mathrm{F}_{2} \mathrm{O}$.

\begin{tabular}{|c|c|c|c|c|c|}
\hline Methods & $\tilde{X}^{1} A_{1}$ & $\tilde{X}^{2} B_{1}$ & ${ }^{2} B_{2}$ & ${ }^{2} A_{1}$ & ${ }^{2} A_{2}$ \\
\hline MP2/cc-pVTZ & $\begin{array}{l}985.5 / 485.5 / \\
917.0\end{array}$ & $\begin{array}{c}1499.2 / 663.3 .3 / \\
1346.9\end{array}$ & $\begin{array}{c}1760.8 / 707.6 / \\
1353.7\end{array}$ & $\begin{array}{c}1109.3 / 485.7 / \\
1811.4\end{array}$ & $\begin{array}{c}946.9 / 323.8 / \\
251.1 \mathrm{i}\end{array}$ \\
\hline B3LYP/cc-pVTZ & $\begin{array}{c}1018.7 / 480.1 / \\
893.8\end{array}$ & $\begin{array}{c}1125.0 / 570.0 / \\
993.5\end{array}$ & $\begin{array}{c}1029.5 / 445.0 / \\
538.9\end{array}$ & $\begin{array}{c}821.9 / 429.7 / \\
385.1\end{array}$ & $\begin{array}{c}959.2 / 424.6 / \\
723.3 \mathrm{i}\end{array}$ \\
\hline QCISD/cc-pVTZ & $\begin{array}{c}1007.5 / 494.0 / \\
951.0\end{array}$ & $\begin{array}{c}1146.3 / 573.8 / \\
1059.0\end{array}$ & $\begin{array}{c}1089.5 / 479.6 / \\
484.3\end{array}$ & $\begin{array}{c}799.0 / 427.3 / \\
6495\end{array}$ & \\
\hline $\begin{array}{l}\operatorname{CASSCF}(7,6) / 6- \\
311+\mathrm{G}(2 \mathrm{~d})\end{array}$ & & & & & $\begin{array}{c}769.2 / 366.0 / \\
575.9\end{array}$ \\
\hline $\begin{array}{l}\operatorname{CASSCF}(7,6) / \mathrm{cc}- \\
\text { pVTZ }\end{array}$ & & & & & $\begin{array}{c}794.6 / 372.1 / \\
573.9\end{array}$ \\
\hline $\begin{array}{l}\operatorname{RCCSD}(T) / \text { aug-cc- } \\
\text { pVTZ }^{\text {a }}\end{array}$ & & & $957.3 / 454.6 / \cdots$ & $862.1 / 414.9 / \cdots$ & $879.2 / 378.9$ \\
\hline $\begin{array}{l}\operatorname{RCCSD}(T) / \text { aug-cc- } \\
\text { pVQZ }^{\text {a }}\end{array}$ & $952.4 / 466.9 / \cdots$ & $\begin{array}{c}1068.3 / 556.7 / \\
\ldots\end{array}$ & & & \\
\hline $\operatorname{CCSD}(T) /$ aug-cc-pVTZ ${ }^{b}$ & $951 / 471 / 865$ & & & & \\
\hline $\operatorname{CCSD}(\mathrm{T}) /$ aug-cc-pVQZ ${ }^{\mathrm{b}}$ & $955 / 474 / 868$ & & & & \\
\hline $\begin{array}{l}\text { Experimental } \\
\text { (fundamental) }\end{array}$ & $\begin{array}{c}924.1504^{\mathrm{c}} / \\
460.56^{\mathrm{d}} / 828.69^{\mathrm{e}}\end{array}$ & $\begin{array}{c}1032^{\mathrm{f}} ; 1010^{\mathrm{g}} ; / \\
1000^{\mathrm{h}} / \cdots / \cdots\end{array}$ & $\cdots / 440^{\mathrm{h}} / \cdots$ & & $900 / 480 \cdots$ \\
\hline $\begin{array}{l}\text { Experimental } \\
{\text { (harmonic })^{\mathrm{i}}}^{\text {har }}\end{array}$ & $\begin{array}{c}941.6 / 467.5 / \\
849.5\end{array}$ & & & & \\
\hline
\end{tabular}

${ }^{\mathrm{a}}$ Fundamental frequencies from the ab initio scans in this work.

${ }^{\mathrm{b}}$ Reference 19.

${ }^{\mathrm{c}}$ Reference 53.

${ }^{\mathrm{d}}$ Reference 54.

${ }^{\mathrm{e}}$ Reference 55 .

${ }^{\mathrm{f}}$ Reference 1 .

${ }^{g}$ Reference 2 .

${ }^{\mathrm{h}}$ This work ( $\pm 20, \pm 30$, and $\pm 50 \mathrm{~cm}^{-1}$ for the ${ }^{2} B_{1},{ }^{2} B_{2}$, and ${ }^{2} A_{2}$ states, respectively).

${ }^{\mathrm{i}}$ Reference 18; estimated from fundamentals and MP2/TZ2PF $\omega_{e} x_{e}$ (from Ref. 17).

CASSCF calculations gave all real frequencies at their respective optimized geometries. The most reliable computed geometrical parameters and fundamental vibrational frequencies of the symmetric stretch and bending modes were, however, obtained from calculations from the scans/PEFs at the highest levels [RCCSD(T)/aug-cc-pVTZ and/or RCCSD(T)/ aug-cc-pVQZ].

\section{B. $A b$ initio calculations: Computed ionization energies}

The computed ionization energies are given in Table IV. First, the first AIE is considered, as reliable experimental values are available. All the computed values obtained in this work agree with the experimental values (including the most reliable experimental value obtained from the present work, $13.11 \mathrm{eV}$ ) to within $0.3 \mathrm{eV}$. If only the $\operatorname{RCCSD}(\mathrm{T})$ values are considered, the agreement is within $0.2 \mathrm{eV}$. At the highest level of calculation $[\operatorname{RCCSD}(\mathrm{T}) / \mathrm{cc}-\mathrm{pV} 5 \mathrm{Z}]$, the computed AIE value agrees with the most reliable experimental value to $\leqslant 0.02 \mathrm{eV}$. The difference between the $\operatorname{RCCSD}(\mathrm{T}) / \mathrm{cc}-$ pVQZ and RCCSD(T)/cc-pV5Z levels is only $0.06 \mathrm{eV}$, suggesting near exhaustion of basis set effects. The CBS limit values obtained from various extrapolation techniques (see Table IV and footnotes $\mathrm{f}, \mathrm{g}, \mathrm{h}$, and i) agree with each other to within $0.03 \mathrm{eV}$, and agree with the most reliable experimental value to less than $0.1 \mathrm{eV}$. All these comparisons support the expectation mentioned in Sec. I that near state-of-the-art quantum chemical calculations on relative energies are reliable to within $0.2 \mathrm{eV}$.

For the order of the low-lying cationic states and the position of the ${ }^{2} A_{2}$ ionic state, a clearer picture is obtained, if the $T_{e} \mathrm{~s}$ (the relative electronic energy of the excited cationic state with respect to the ground cationic state), rather than the AIEs, are considered. From Table V, the consistency in the computed $T_{e}$ values of the low-lying states of $\mathrm{F}_{2} \mathrm{O}^{+}$obtained at various levels of calculation is remarkably high. Therefore, it can be concluded that the reliability of these values is beyond any reasonable doubt. The order of the low-lying states of $\mathrm{F}_{2} \mathrm{O}^{+}$is conclusively ${ }^{2} B_{1},{ }^{2} B_{2},{ }^{2} A_{1}$, and ${ }^{2} A_{2}$, which is the same as for $\mathrm{Cl}_{2} \mathrm{O}^{+}$(see Refs. 10 and 12), but different from the original assignments of Cornford and co-workers and Brundle and co-workers ${ }^{2}$ in the early PE studies. From the present systematic theoretical investigation on the computed IEs, it can be concluded that it would be very unlikely for this order to be changed with any further improvement in the level of calculation.

Regarding the position of the ${ }^{2} A_{2}$ state, it can be seen from the RCCSD(T) calculations in Table $\mathrm{V}$ that the range of the computed $T_{e}$ values is from 3.4 to $3.6 \mathrm{eV}$. Combining the experimental AIE of the first band with the computed $T_{e}$ values, the AIE values to the ${ }^{2} A_{2}$ state range from 16.5 to $16.7 \mathrm{eV}$. In addition, the differences between the computed $T_{e}$ values of the ${ }^{2} A_{1}$ and ${ }^{2} A_{2}$ states obtained at various levels 
TABLE IV. The observed and calculated adiabatic and vertical ionization energies (AIE/VIE in eV) to the four lowest energy cationic states $\left(\tilde{X}^{2} B_{1},{ }^{2} B_{2},{ }^{2} A_{1}\right.$, and $\left.{ }^{2} A_{2}\right)$ in the He I PE spectrum of $\mathrm{F}_{2} \mathrm{O}$.

\begin{tabular}{|c|c|c|c|c|}
\hline Methods & $\tilde{X}^{2} B_{1}$ & ${ }^{2} B_{2}$ & ${ }^{2} A_{1}$ & ${ }^{2} A_{2}$ \\
\hline \multirow[t]{3}{*}{ Experimental values } & $3.13 / 13.26^{\mathrm{a}}$ & $\cdots / 16.47^{\mathrm{a}}$ & $\cdots / 16.17^{\mathrm{a}}$ & $\cdots / 18.68^{\mathrm{a}}$ \\
\hline & $13.11 / 13.25^{\mathrm{b}}$ & $\cdots / 16.44^{b}$ & $15.74 / 16.10^{\mathrm{b}}$ & $17.9 / 18.50^{\mathrm{b}}$ \\
\hline & $13.109 / 13.233^{c}$ & $15.83 / 16.11^{\mathrm{d}}$ & & $16.44 / 16.44^{\mathrm{e}}$ \\
\hline MP2/cc-pVTZ & $13.42 / \cdots$ & $16.28 / \cdots$ & $16.84 / \cdots$ & $16.42 / \cdots$ \\
\hline QCISD/cc-pVTZ & $12.84 / \cdots$ & $15.76 / \cdots$ & $15.99 / \ldots$ & \\
\hline RCCSD/aug-cc-pVQZ & $\cdots / 13.40$ & $\cdots / 16.28$ & $\cdots / 16.74$ & \\
\hline \multicolumn{5}{|l|}{ //QCISD/cc-pVTZ } \\
\hline RCCSD(T)cc-pVTZ & $12.93 / \cdots$ & $15.72 / \cdots$ & $16.19 / \cdots$ & $16.46 / \cdots$ \\
\hline RCCSD(T)/cc-pVQZ & $13.07 / \cdots$ & $15.78 / \cdots$ & $16.36 / \cdots$ & $16.63 / \cdots$ \\
\hline $\operatorname{RCCSD}(\mathrm{T}) / \mathrm{cc}-\mathrm{pV} 5 \mathrm{Z} / /$ & $13.13 / \cdots$ & $15.85 / \cdots$ & $16.43 / \cdots$ & $16.71 / \cdots$ \\
\hline \multicolumn{5}{|l|}{ RCCSD(T)cc-pVQZ } \\
\hline MRCI+D/cc-pVQZ// & $12.87 / 13.07$ & $15.59 / 15.97$ & $15.99 / 16.18$ & $16.26 / 16.41$ \\
\hline \multicolumn{5}{|l|}{ RCCSD(T)/cc-pVQZ } \\
\hline Extrapol. VTZ/VQZ & $13.17 / \cdots$ & $15.89 / \cdots$ & $16.48 / \cdots$ & $16.76 / \cdots$ \\
\hline VQZ/V5Z & $13.20 / \cdots$ & $15.91 / \cdots$ & $16.51 / \cdots$ & $16.78 / \cdots$ \\
\hline Extrapol. VDZ/VTZ & $13.11 / \cdots$ & $15.86 / \cdots$ & $16.41 / \cdots$ & $16.72 / \cdots$ \\
\hline \multirow[t]{2}{*}{ Extrapol. VDZ-V5Z } & $13.19 / \cdots$ & $15.79 / \cdots$ & $16.48 / \cdots$ & $16.75 / \cdots$ \\
\hline & $13.18 / \cdots$ & $15.90 / \cdots$ & $16.48 / \cdots$ & $16.76 / \cdots$ \\
\hline Extrapol. VDZ-V5Z & $13.20 / \cdots$ & $15.90 / \cdots$ & $16.49 / \cdots$ & $16.75 / \cdots$ \\
\hline Spectral simulation ${ }^{\mathrm{j}}$ & & $15.71 / 16.11$ & $16.17 / 16.40$ & $16.44 / 16.54$ \\
\hline
\end{tabular}

${ }^{\mathrm{a}}$ Reference 1 .

${ }^{\mathrm{b}}$ Reference 2 .

${ }^{\mathrm{c}}$ This work $( \pm 0.010 \mathrm{eV})$; see text.

${ }^{\mathrm{d}}$ This work $( \pm 0.02 \mathrm{eV})$; the AIE value given here is the position of the first identifiable peak (see text).

${ }^{\mathrm{e}}$ This work $( \pm 0.02 \mathrm{eV})$; see text.

${ }^{\mathrm{f}}$ Two point extrapolation (see Ref. 36): $E_{\lim }=X^{\alpha} /\left(X^{\alpha}-(X-1)^{\alpha}\right) E_{X}-(X-1)^{\alpha} /\left(X^{\alpha}-(X-1)^{\alpha}\right) E_{X-1}$; data from the RCCSD(T)cc-pVTZ (i.e., VTZ) and RCCSD(T)/cc-pVQZ (i.e., VQZ) calculations etc. (similar for the rest in the Table).

${ }^{\mathrm{g}}$ Two point extrapolation (VDZ and VTZ data; see Ref. 37): $E_{\lim }=3^{\alpha} /\left(3^{\alpha}-2^{\alpha}\right) E_{3}^{\mathrm{HF}}-2^{\alpha} /\left(3^{\alpha}-2^{\alpha}\right) E_{2}^{\mathrm{HF}}$ $+3^{\beta} /\left(3^{\beta}-2^{\beta}\right) E_{3}^{\text {cor }}-2^{\beta} /\left(3^{\beta}-2^{\beta}\right) E_{2}^{\text {cor }}$.

${ }^{\text {h}}$ Energies fitted to (see Ref. 38): $E_{\mathrm{SCF}}(X)=E_{\mathrm{SCF}}+B e^{-\alpha x}$ and $E_{\text {corr }}(X)=E_{\text {corr }}+B^{\prime} X^{-3}$.

i Energies fitted to (see Ref. 39): $E(X)=E_{\lim }+F(X)$, where $F(X)=B e^{-\alpha x}$.

${ }^{\mathrm{j}}$ See text.

of calculation (Table V) are remarkably consistent. Excluding the extrapolated CBS values, obtained using the twopoint formula of Ref. 37 (which give a separation of 0.31 $\mathrm{eV})$, the average computed $\mathrm{AIE} / T_{e}$ separation between the ${ }^{2} A_{1}$ and ${ }^{2} A_{2}$ state is $0.27 \pm 0.02 \mathrm{eV}$. The CASSCF/MRCI separation is $0.27 \mathrm{eV}$, in excellent agreement with

TABLE V. The computed relative electronic energies, $T_{e}$ (in $\mathrm{eV}$ ), of the low-lying cationic states of $\mathrm{F}_{2} \mathrm{O}^{+}$(with respect to the $\tilde{X}^{2} B_{1}$ state).

\begin{tabular}{lccc}
\hline \hline \multicolumn{1}{c}{ Method } & ${ }^{2} B_{2}$ & ${ }^{2} A_{1}$ & ${ }^{2} A_{2}$ \\
\hline RCCSD(T)/cc-pVTZ & 2.79 & 3.26 & 3.53 \\
RCCSD(T)/cc-pVQZ & 2.71 & 3.29 & 3.56 \\
RCCSD(T)/ccpV5Z// & 2.72 & 3.30 & 3.58 \\
RCCSD(T)/cc-pVQZ & & & \\
MRCI+D/cc-pVQZ// & 2.72 & 3.12 & 3.39 \\
RCCSD(T)/cc-pVQZ & & & \\
Extrapol. VTZ/VQZ & 2.72 & 3.31 & 3.59 \\
VQZ/V5Z & 2.71 & 3.31 & 3.58 \\
Extrapol. VDZ/VTZ & 2.75 & 3.30 & 3.61 \\
Extrapol. VDZ-V5Z & 2.60 & 3.29 & 3.57 \\
VTZ-V5Z $^{\mathrm{c}}$ & 2.72 & 3.30 & 3.58 \\
Extrapol. VDZ-V5Z & & 3.29 & 3.55 \\
\hline \hline
\end{tabular}

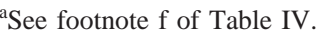

${ }^{\mathrm{b}}$ See footnote $\mathrm{g}$ of Table IV

${ }^{\mathrm{c}}$ See footnote $\mathrm{h}$ of Table IV.

${ }^{\mathrm{d}}$ See footnote i of Table IV.
RCCSD(T) values and most of the CBS values. Based on all these highly consistent computed results, it can be concluded that the ${ }^{2} A_{1}$ and ${ }^{2} A_{2}$ states should be very close, with a separation of $\leqslant 0.3 \mathrm{eV}$. This conclusion clearly favors the alternative $\mathrm{PE}$ assignment suggested by Brundle and co-workers ${ }^{2}$ that the ionization leading to the ${ }^{2} A_{2}$ state has to be assigned together with the ionizations leading to the ${ }^{2} B_{2}$ and ${ }^{2} A_{1}$ states in the second and third $\mathrm{PE}$ bands of $\mathrm{F}_{2} \mathrm{O}$. However, the detailed assignments of these two observed bands and the precise AIE/VIE positions of the three cationic states, which contribute to these two experimental PE bands, could not be deduced unambiguously from the observed spectrum without the aid of spectral simulations, which will be discussed later. It is just noted that the $\triangle$ SCF AIE values obtained with the different basis sets used in this work have also been examined. It is found that the large differences in the computed correlation energies between the neutral ground state and the cationic ${ }^{2} A_{2}$ state have caused the correlated AIEs to have significantly lower values than the corresponding $\triangle \mathrm{SCF}$ values. Higher computed IE values from previous calculations for the ${ }^{2} A_{2}$ state of $\mathrm{F}_{2} \mathrm{O}^{+}$(for examples, Refs. 2, 3, and 7; see Table I) are almost certainly due to a lack of inclusion of sufficient electron correlation in the calculations. 
TABLE VI. The calculated anharmonic PEFs (in units of Hartree $\AA^{-m} \operatorname{rad}^{-n}$ for $m$ stretching and $n$ bending coordinates) for the neutral ground state $\left(\widetilde{X}^{1} A_{1}\right)$ and cationic states $\left(\tilde{X}^{2} B_{1},{ }^{2} B_{2},{ }^{2} A_{1}\right.$, and $\left.{ }^{2} A_{2}\right)$ of $\mathrm{F}_{2} \mathrm{O}$.

\begin{tabular}{rrrrrr}
\hline \hline$C_{i j}{ }^{\mathrm{a}}$ & \multicolumn{1}{c}{$\tilde{X}^{1} A_{1}{ }^{\mathrm{b}}$} & \multicolumn{1}{c}{$\tilde{X}^{2} B_{1}{ }^{\mathrm{b}}$} & \multicolumn{1}{c}{${ }^{2}{ }^{2} B_{2}{ }^{\mathrm{c}}$} & \multicolumn{1}{c}{${ }^{2}{ }^{2}{ }_{1}{ }^{\mathrm{c}}$} & \multicolumn{1}{c}{$C^{2} A_{2}{ }^{\mathrm{c}}$} \\
\hline $\mathbf{2 0}$ & 0.5722 & 0.7628 & 0.5545 & 0.6165 & 0.4712 \\
$\mathbf{1 1}$ & 0.0630 & 0.1005 & 0.2042 & 0.1081 & 0.0436 \\
$\mathbf{0 2}$ & 0.1683 & 0.2000 & 0.1927 & 0.0996 & 0.1250 \\
$\mathbf{3 0}$ & -0.9291 & -1.3654 & -0.8852 & -1.0596 & -0.7307 \\
$\mathbf{2 1}$ & -0.2450 & -0.3649 & -0.5370 & -0.2215 & -0.1763 \\
$\mathbf{1 2}$ & -0.4573 & -0.5425 & -0.7579 & -0.2653 & -0.3924 \\
$\mathbf{0 3}$ & 0.0405 & 0.0614 & -0.1597 & -0.0219 & 0.0191 \\
$\mathbf{4 0}$ & 0.8944 & 1.4570 & 0.7510 & 0.9484 & 0.5865 \\
$\mathbf{3 1}$ & 0.4298 & 0.5556 & 0.7623 & 0.2837 & 0.3495 \\
$\mathbf{2 2}$ & 0.5696 & 0.6467 & 0.8996 & 0.3399 & 0.5480 \\
$\mathbf{1 3}$ & 0.0328 & 0.0008 & 0.2398 & 0.1019 & 0.0670 \\
$\mathbf{0 4}$ & 0.1922 & 0.2293 & 0.2822 & 0.1012 & 0.1717 \\
$\mathbf{5 0}$ & -0.7661 & -1.2534 & & & \\
$\mathbf{0 5}$ & 0.2009 & 0.3029 & 1.0120 & 0.0455 & 0.1584 \\
$\mathbf{6 0}$ & 0.4288 & 0.6481 & & & \\
$\mathbf{0 6}$ & 0.1404 & 0.2155 & 1.1904 & 0.0474 & 0.1013 \\
$\mathbf{r}_{\mathbf{e}}$ & 1.4052 & 1.3185 & 1.4441 & 1.3686 & 1.4690 \\
$\theta_{\mathbf{e}}$ & 1.7975 & 1.8714 & 1.4345 & 2.0699 & 1.7099 \\
$\alpha$ & 0.0703 & 0.0706 & 0.1868 & -0.1193 & 0.1047 \\
$\mathbf{V}_{\mathbf{e}}$ & -274.4461 & -273.9632 & -273.7956 & -273.7732 & -273.7648 \\
\hline \hline
\end{tabular}

${ }^{a}$ See text for the definitions of $C_{i j}, r_{e}, \alpha$, and $V_{e}$.

${ }^{\mathrm{b}}$ Employing RCCSD(T)/aug-cc-pVQZ energy points (see text).

${ }^{c}$ Employing RCCSD(T)/aug-cc-pVTZ energy points (see text).

\section{The fitted PEFs and the anharmonic vibrational wave functions}

The $C_{i j}, \alpha, r_{e}$, and $\theta_{e}$ values obtained from the fitting are given in Table VI for all the electronic states studied. The root-mean-square deviations in the fits are smaller than 11 $\mathrm{cm}^{-1}$ in all the PEFs reported here. For the $\tilde{X}^{1} A_{1}$ state of neutral $\mathrm{F}_{2} \mathrm{O}$, the anharmonic force field at the $\operatorname{CCSD}(\mathrm{T})$ level (quadratic force field from CCSD(T)/cc-pVQZ, cubic, and quartic force field from CCSD(T)/cc-pVTZ calculations) has been reported by Breidung and co-worker. ${ }^{19}$ Variational calculation of vibrational wave functions was also performed employing the best PEF from Ref. 19. A basis size limit of $v_{1}, v_{2} \leqslant 10$ and a further constraint of $v_{1}+v_{2} \leqslant 10$ was imposed, when only the two symmetric vibrational modes were considered. When all three vibrational modes are considered, a basis limit of $v_{1}, v_{2}, v_{3} \leqslant 10$ with a constraint of $v_{1}+v_{2}$ $+v_{3} \leqslant 10$ was imposed. The computed vibrational energies thus obtained are compared with those obtained using the PEF of the present study for the neutral ground state of $\mathrm{F}_{2} \mathrm{O}$, and this comparison is shown in Table VII. The agreement among the computed vibrational energies (and with available experimental values) is generally very good. The effect of including the asymmetric mode, $\nu_{3}$, in the variational calculation on the computed vibrational energies of the two symmetric modes $\left(\nu_{1}\right.$ and $\left.\nu_{2}\right)$ seems small. The present PEF for the ground state of $\mathrm{F}_{2} \mathrm{O}$ may be considered to be of a slightly higher quality than those reported by Breidung and co-workers, ${ }^{19}$ as the basis set used here (aug-cc-pVQZ), which includes diffuse valence and polarization functions, is of higher quality than those (cc-pVTZ and cc-pVQZ) used in Ref. 19.

It should be noted that for the neutral ground state of
TABLE VII. The observed and calculated vibrational term values (with respect to the ground vibrational state in $\mathrm{cm}^{-1}$ ) of $\tilde{X}^{1} A_{1}$ state of $\mathrm{F}_{2} \mathrm{O}$.

\begin{tabular}{ccccc}
\hline \hline$v_{1} v_{2} v_{3}{ }^{\mathrm{a}}$ & $\mathrm{Obs}^{\mathrm{b}}$ & $\mathrm{Calc}^{\mathrm{c}}$ & $\begin{array}{c}\text { Breidung } \\
\text { and co-workers }^{\mathrm{d}}\end{array}$ & $\begin{array}{c}\text { Breidung } \\
\text { and co-workers }\end{array}$ \\
\hline 010 & 460.56 & 464.1 & 461.7 & 465.1 \\
020 & 916.04 & 924.7 & 928.1 & 925.9 \\
100 & 928.07 & 943.2 & 943.9 & 937.6 \\
110 & & 1382.7 & 1388.5 & 1384.4 \\
030 & & 1406.8 & 1408.4 & 1403.1 \\
040 & & 1838.1 & 1846.6 & 1840.1 \\
120 & & 1864.7 & 1868.9 & 1859.5 \\
200 & & 1878.4 & 1880.2 & 1871.2 \\
050 & & 2291.4 & 2302.7 & 2294.5 \\
130 & & 2320.6 & 2327.5 & 2317.6 \\
210 & & 2342.4 & 2345.4 & 2337.6 \\
\hline \hline
\end{tabular}

${ }^{a}$ The vibrational quantum numbers, $v_{1}, v_{2}$, and $v_{3}$, correspond to the symmetric stretching, bending and asymmetric stretching modes, respectively. ${ }^{b}$ References 53, 54, and 55.

${ }^{c}$ Employing the RCCSD(T)/aug-cc-pVQZ PEF of this work; see text for details.

"Employing the "best" PEF of Ref. 19 with the asymmetric mode ignored in the basis set (see text).

'Employing the "best" PEF of Ref. 19 (see text).

$\mathrm{F}_{2} \mathrm{O}$ significant mixing of harmonic basis functions of the stretching and bending modes occurs in the computed anharmonic vibrational levels. This is expected, as the $2 \nu_{2}$ and $\nu_{1}$ levels (for $\left.v_{2}=v_{1}\right)$ have close vibrational energies $\left(2 \nu_{2}\right.$ $\approx \nu_{1}$ ) particularly for the $\tilde{X}^{1} A_{1}$ state of neutral $\mathrm{F}_{2} \mathrm{O}$. The labels given in Table VII are based on the computed largest coefficients of the harmonic basis function in the respective anharmonic vibrational wave functions. However, for highly excited vibrational levels, mixing could become so strong that their labels cannot be determined unambiguously. Nevertheless, for all the cationic states considered here, mixing between the symmetric stretching and bending mode is negligible.

The computed fundamental frequencies obtained from the PEFs of this work are given in Table III. In general, they are in closer agreement with available experimental values than the computed harmonic frequencies, as expected.

\section{Spectral simulation: First PE band}

The observed first band in the $\mathrm{He}$ I PE spectrum of $\mathrm{F}_{2} \mathrm{O}$ from the present study and its simulations employing the anharmonic and harmonic FCF methods are shown in Figs. 1(a), 1(b) and 1(c), respectively. The measured AIE/VIE and vibrational spacings of this band are given in Tables IV and III, respectively. They are in excellent agreement with previously reported experimental values, but with smaller uncertainties, as the present spectrum is of a higher quality. The "hot band" associated with the first PE band is clearly observed. The observed vibrational components, which belong to the symmetric stretching progression of the cation, are resolved to the baseline. It is clear that excitation of the bending mode is not involved.

From spectral simulation, a temperature of $300 \mathrm{~K}$ is sufficient to produce a simulated hot band in the symmetric stretching mode of the neutral (assuming a Boltzmann distri- 

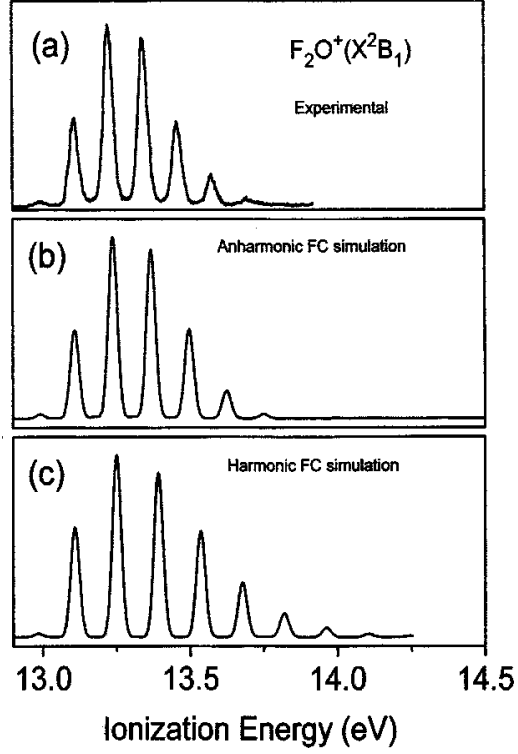

FIG. 1. The first band in the He I PE spectrum of $\mathrm{F}_{2} \mathrm{O}$ : (a) observed (from this work), (b) simulated (with a FWHM of $35 \mathrm{meV}$ ) employing AN-FCFs, and (c) simulated with harmonic FCFs (see text).

bution of vibrational levels in the initial state), which matches well with that in the observed spectrum. Therefore, all the simulations performed in this work have assumed an initial state Boltzmann distribution at $300 \mathrm{~K}$. The simulated spectra shown were obtained after applying the IFCA procedure. The corresponding IFCA ionic state geometrical parameters are given in Table II. From Fig. 1, it is clear that the simulated spectrum with harmonic FCFs gives a symmetric stretching progression which is too long when compared with the observed spectrum. Nevertheless, it is pleasing that the simulated spectrum with anharmonic FCFs matches the observed spectrum almost exactly.

The IFCA geometrical parameters obtained from the anharmonic simulation for the cationic ground state are $R(\mathrm{FO})=1.323 \pm 0.002 \AA$ and $<\mathrm{FOF}=107.3 \pm 0.2^{\circ}$. The computed FCFs are more sensitive to the geometry changes upon ionization than the quoted uncertainties of the IFCA geometrical parameters. The estimated uncertainties given have taken into account factors such as the approximations and assumptions involved in the theoretical model and experimental limitations from the observed spectra (e.g., the nonobservance of the bending mode in the first PE band). Further details of how the uncertainties were obtained are given in Ref. 52. It is noted here that with the harmonic simulation, despite various attempts, it was not possible to obtain a good match across the whole stretching progression of the PE band. The IFCA geometry given in Table II with the harmonic method was obtained mainly by matching the relative intensities of the most intense components in the low IE region, and assuming that the relative intensities of the bending mode components are negligibly small. It seems clear that the harmonic oscillator model is inadequate for the ionization to the $\tilde{X}^{2} B_{1}$ state. The anharmonic simulation is more convincing and the IFCA ionic state geometry derived from these simulations is the most reliable.

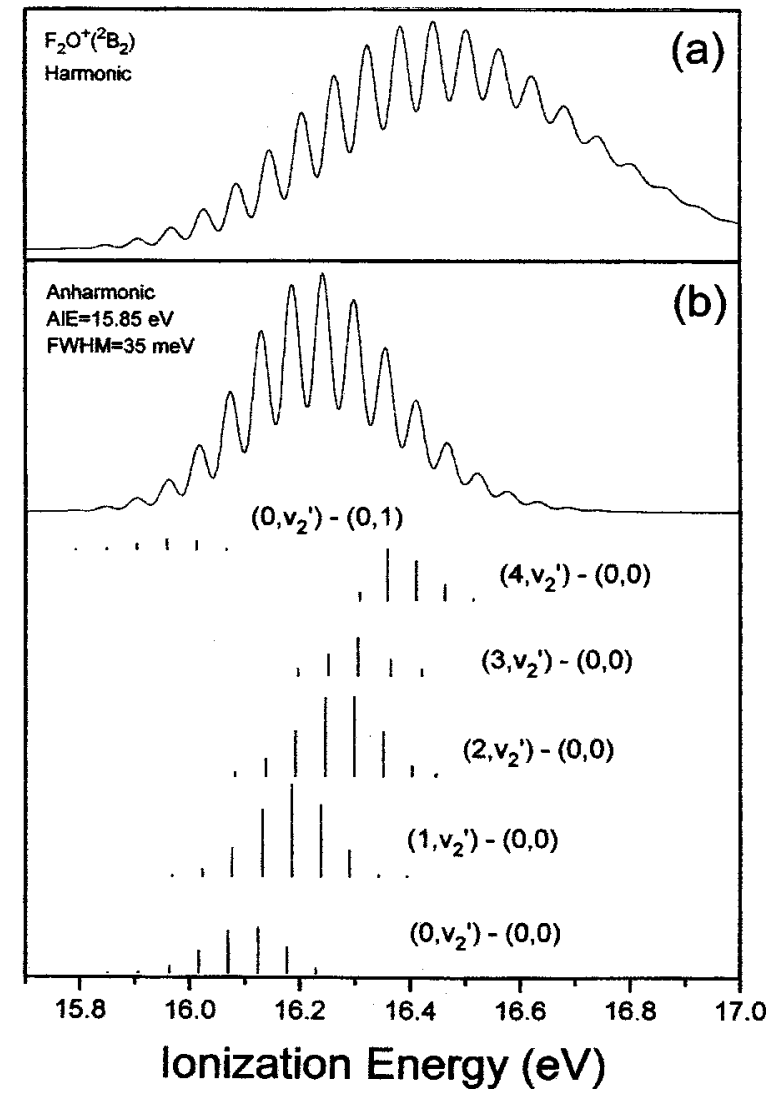

FIG. 2. The simulated spectra (with a FWHM of $35 \mathrm{meV}$ ) of the $\mathrm{F}_{2} \mathrm{O}^{+}\left({ }^{2} B_{2}\right) \leftarrow \mathrm{F}_{2} \mathrm{O}\left(\tilde{X}^{1} A_{1}\right)$ ionization, obtained using (a) harmonic and (b) AN-FCFs (bar diagrams showing the computed FCFs in the symmetric stretching and bending modes).

\section{E. Spectral simulation: Second and third PE bands}

Before the simulated spectra for the ionizations leading to the ${ }^{2} B_{2},{ }^{2} A_{1}$, and ${ }^{2} A_{2}$ cationic states are discussed (Figs. $2-4)$, the He I PE spectrum obtained in the present study deserves some attention. The second and third bands in the $\mathrm{He}$ I PE spectrum of $\mathrm{F}_{2} \mathrm{O}$ obtained in the present investigation are shown in Fig. 5 (top spectrum). Partially resolved vibrational structure is clearly observed on the low IE side of the second band, and the high IE side of the third band. For the former, a single vibrational progression with a number of components can be identified [labeled (a) in Fig. 5]. The first observable component has a measured IE of $15.83 \mathrm{eV}$ and at least five components are observed. However, the vibrational structure becomes diffuse in a region beyond the maximum of the second band (a VIE of $16.11 \mathrm{eV}$ ). For the vibrational structure of the third band, two vibrational progressions can be identified [labeled (b) and (c) in Fig. 5]. The AIE and VIE positions of this band appear to coincide at the band maximum of $16.44 \mathrm{eV}$. The measured vibrational spacings in the second and third bands are reported here, with their values given in Table III. These values are in reasonably good agreement with the computed vibrational frequencies of the ${ }^{2} B_{2}$ and ${ }^{2} A_{2}$ ionic states, respectively (see Table III). In addition, between the two band maxima, some poorly resolved vibrational structure can be seen, though reliable vibrational spacings could not be obtained. The measured IE positions 


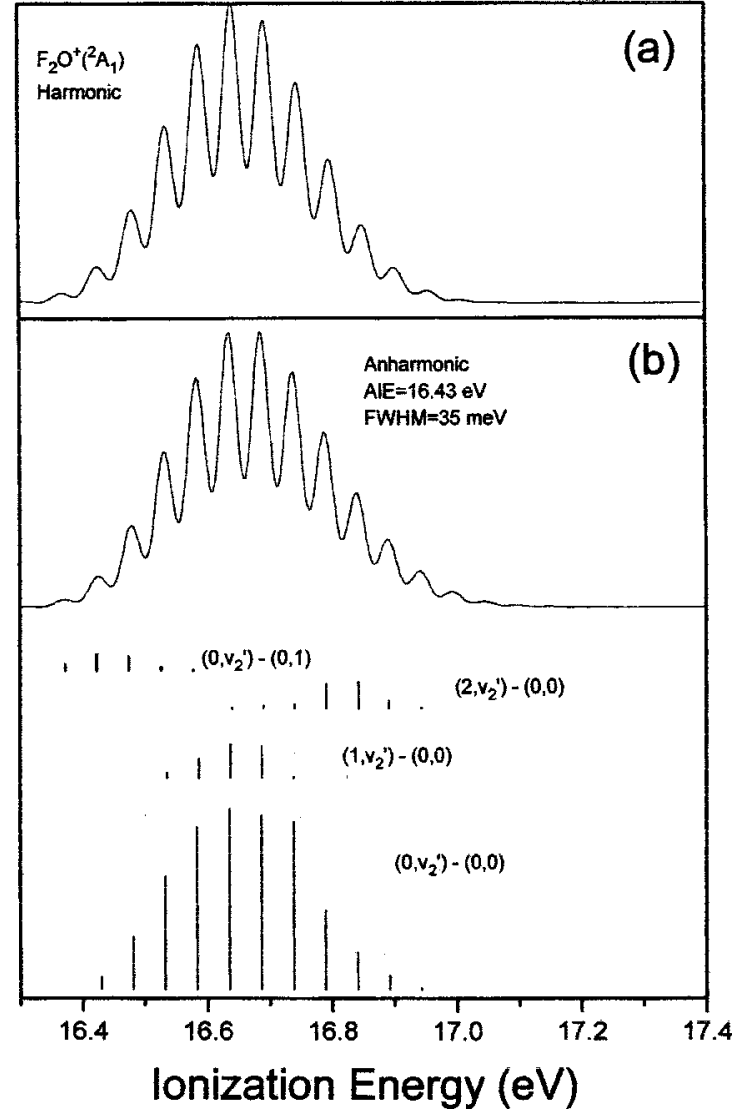

FIG. 3. The simulated spectra (with a FWHM of $35 \mathrm{meV}$ ) of the $\mathrm{F}_{2} \mathrm{O}^{+}\left({ }^{2} A_{1}\right) \leftarrow \mathrm{F}_{2} \mathrm{O}\left(\tilde{X}^{1} A_{1}\right)$ ionization, obtained using (a) harmonic and (b) AN-FCFs (bar diagrams showing the computed FCFs in the symmetric stretching and bending modes).

of the onset of the second band and the band maxima of the second and third bands are in good agreement with previously reported values (though the assignments are different) and are given in Table IV.

The simulated spectra for ionizations to the ${ }^{2} B_{2},{ }^{2} A_{1}$, and ${ }^{2} A_{2}$ states are shown individually in Figs. 2-4, respectively, with bar diagrams showing the computed FCFs. It can be seen that the simulated spectra employing the harmonic and anharmonic FC models for ionizations to the ${ }^{2} A_{1}$ and ${ }^{2} A_{2}$ ionic states are almost identical (Figs. 3 and 4). However, for the ionization to the ${ }^{2} B_{2}$ state, the harmonic simulation has a significantly longer progression than the anharmonic one (Fig. 2). From here onward, only the anharmonic simulations will be considered, as they should be more reliable. For the $\mathrm{F}_{2} \mathrm{O}^{+2} A_{2} \leftarrow \mathrm{F}_{2} \mathrm{O} \tilde{X}^{1} A_{1}$ ionization, an IFCA simulated spectrum is also shown in Fig. 4(c) and this will be discussed next.

Regarding the comparison between the simulated and observed spectra, ionization to all three states, namely the ${ }^{2} B_{2},{ }^{2} A_{1}$, and ${ }^{2} A_{2}$ states, have to be considered together, as they overlap to form the observed second and third PE bands. Figure 5(c) shows the simulated composite spectrum, based on the highest levels of calculations [i.e., combining Figs. 2(b), 3(b), and 4(b)]. Comparing this "purely" theoretical simulation with the observed spectrum, it seems clear that the observed second and third bands are mainly due to

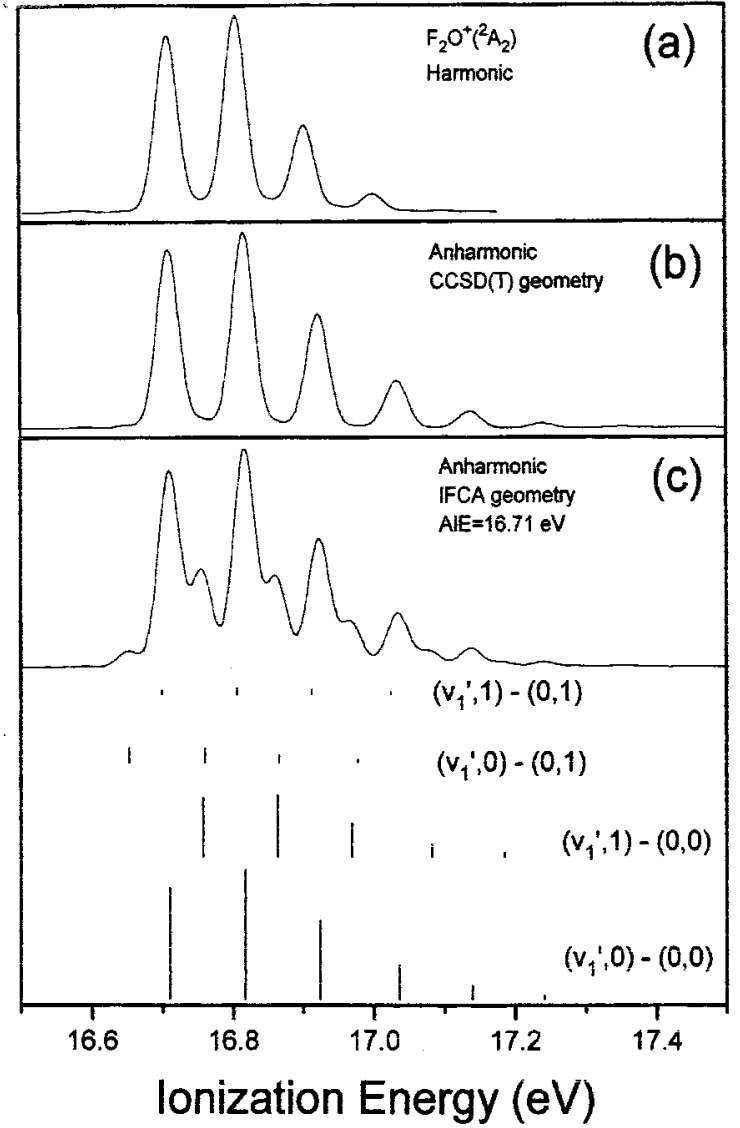

FIG. 4. The simulated spectra (with a FWHM of $35 \mathrm{meV}$ ) of the $\mathrm{F}_{2} \mathrm{O}^{+}\left({ }^{2} A_{2}\right) \leftarrow \mathrm{F}_{2} \mathrm{O}\left(\tilde{X}^{1} A_{1}\right)$ ionization, obtained using (a) harmonic FCFs, (b) AN-FCFs with the RCCSD(T)/cc-pVQZ geometrical parameters of the ${ }^{2} \mathrm{~A}_{2}$ state, and (c) AN-FCFs with the IFCA geometry of $R(\mathrm{OF})=1.460 \AA$ and $<\mathrm{FOF}=95.0^{\circ}$ (bar diagrams showing the computed FCFs in the symmetric stretch and bending modes; see text).

ionization leading to the ${ }^{2} B_{2}$ and ${ }^{2} A_{2}$ states respectively, and the ionization to the ${ }^{2} A_{1}$ state would be in between the second and third bands. These assignments are similar to those in the He I PE spectrum of $\mathrm{Cl}_{2} \mathrm{O}$ (see Refs. 10 and 12).

Figure 5(b) shows the simulation that matches best with the experimental spectrum with the following empirical adjustments. The simulated band maximum of ionization to the ${ }^{2} B_{2}$ state is set to align with the band maximum of the observed second band $(16.11 \mathrm{eV})$. The AIE position of ionization to the ${ }^{2} A_{2}$ simulation is set to the band maximum of the observed third PE band $(16.44 \mathrm{eV})$. The highly consistent computed $\mathrm{AIE} / T_{e}$ separation of $0.27 \mathrm{eV}$ (see Sec. IVE on the IEs) between the ${ }^{2} A_{1}$ and ${ }^{2} A_{2}$ states is employed in the simulation for setting the position of the ${ }^{2} A_{1}$ state. The IFCA procedure was carried out only for the ionization leading to the ${ }^{2} A_{2}$ state. The IFCA geometrical parameters of $R(\mathrm{OF})$ $=1.460 \AA$ and $\angle \mathrm{FOF}=95.0^{\circ}$ for the ${ }^{2} A_{2}$ state were found to give the simulation which matched best with the experimental envelope.

The following points should be noted regarding the simulation which matches best for the second and third bands. First, the IFCA procedure has not been carried out on the ${ }^{2} B_{2} \leftarrow \tilde{X}^{1} A_{1}$ and the ${ }^{2} A_{1} \leftarrow \tilde{X}^{1} A_{1}$ ionizations, because their AIE positions cannot be identified unambiguously from 

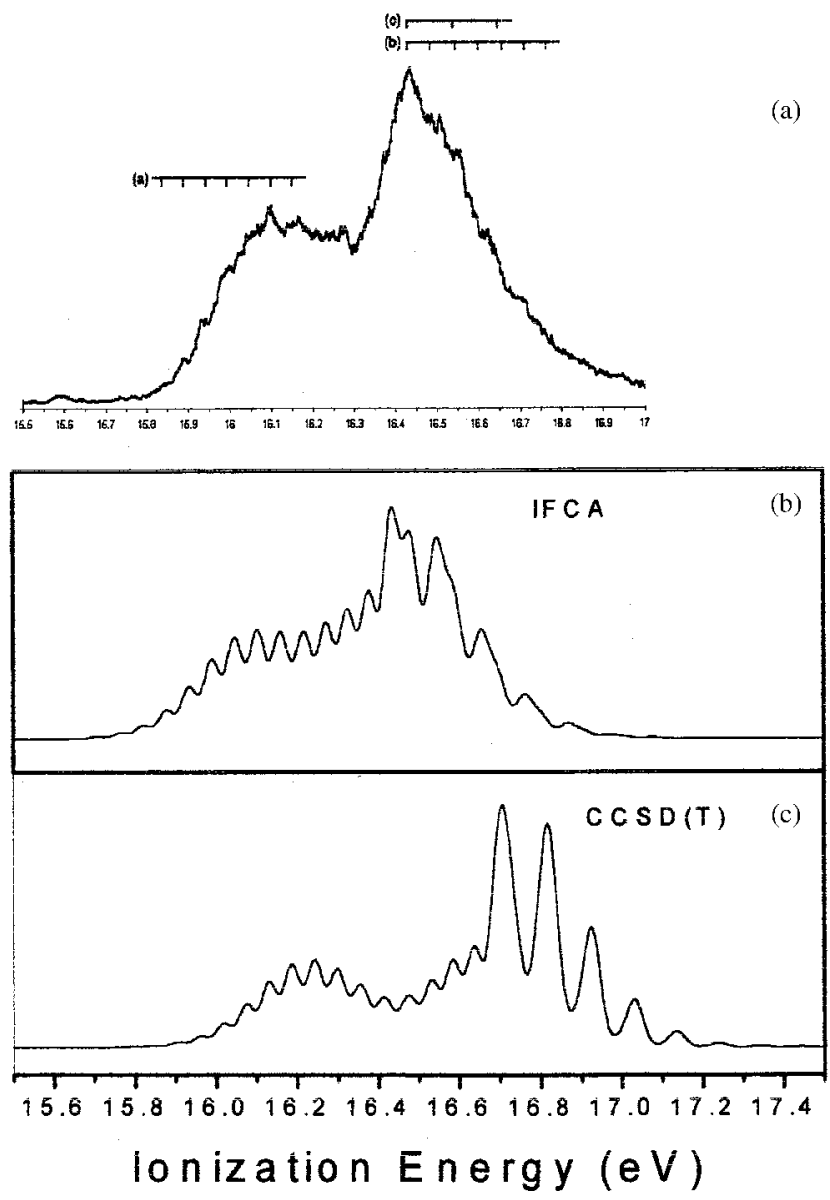

FIG. 5. The second and third bands in the He I PE spectrum of $\mathrm{F}_{2} \mathrm{O}$ : (top) experimental spectrum from this work [(a), (b), and (c) are labels of the three vibrational progressions identified from the experimental spectrum (see text for details)]; (middle) simulated spectrum employing AN-FCFs with some empirical adjustments of band positions and the IFCA geometry of the ${ }^{2} A_{2}$ ionic state [Fig. 4(c)] see text for details]; (bottom) simulated spectrum employing AN-FCFs with RCCSD(T)/cc-pV5Z//RCCSD(T)/ccpVQZ AIEs and RCCSD(T)/cc-pVQZ geometrical parameters [i.e., combining Figs. 2(b), 3(b), and 4(b)].

the observed spectrum. (There is, thus, no way to lock the simulated spectrum to the observed one.) If the IFCA procedure were to be carried out on these envelopes (as even the best $a b$ initio geometry is likely to differ from the true one), the simulated band maximum (i.e., the VIE position) and the position of the first observable vibrational component would change. These changes may help to obtain a better match with the observed spectrum without adjusting the AIE and/or VIE positions, as previously described. Nevertheless, it is felt that slight adjustments on the computed AIE and/or VIE values involve less arbitrariness than carrying out the IFCA procedure on bands associated with ionizations to the ${ }^{2} B_{2}$ and ${ }^{2} A_{1}$ states, where the vibrational structure is only partially resolved $\left({ }^{2} B_{2}\right.$ band) or not resolved $\left({ }^{2} A_{1}\right)$.

Second, regarding carrying out the IFCA procedure on the $\mathrm{F}_{2} \mathrm{O}^{+}\left({ }^{2} A_{2}\right) \leftarrow \mathrm{F}_{2} \mathrm{O}\left(\tilde{X}^{1} A_{1}\right)$ ionization, it was found that employing the geometry obtained from the highest level of calculations [RCCSD(T)/cc-pVQZ], the simulated spectrum could not give a reasonable match with the observed spectrum (the third band). With the RCCSD(T)/cc-pVQZ geometry for the ${ }^{2} A_{2}$ ionic state, the bending mode is very weak and hence the stretching progression should be well resolved [Fig. 4(b)]. However, the vibrational structure observed on the high IE side of the third band could not be well resolved [Fig. 5(a)]. This is despite the fact that the resolution FWHM, as judged from other resolved features observed elsewhere in the spectrum (e.g., argon), is better than 30 meV. Clearly, the bending mode must have been invoked in the ionization process in order to produce the observed vibrational structure. From Table II, it is clear that the magnitudes of the computed geometrical parameters for the ${ }^{2} A_{2}$ ionic state are very sensitive to the level of calculations, as just mentioned briefly. The computed bond lengths range from ca. 1.44 to $1.51 \AA$ and the bond angles, from $94.70^{\circ}$ to $104.9^{\circ}$. The IFCA geometrical parameters used for the ${ }^{2} A_{2}$ state to produce the best match with the observed third PE band are within the ranges of the computed ones. It seems that the RCCSD(T) bond length and CASSCF bond angle give the best combination. The IFCA geometrical parameters for $\mathrm{F}_{2} \mathrm{O}^{+}\left({ }^{2} A_{2}\right)$ are recommended as $R(\mathrm{OF})=1.460$ $\pm 0.050 \AA$ and $\angle(\mathrm{FOF})=95.0 \pm 0.5^{\circ}$. The computed envelope with these ionic state IFCA parameters is shown in Fig. 4(c).

\section{CONCLUDING REMARKS}

Summarizing, the $\mathrm{He}$ I PE spectrum of $\mathrm{F}_{2} \mathrm{O}$ has been recorded with a better resolution than previously reported. New vibrational structure associated with the second and third PE bands has been observed. The order and positions of the lowest four states of $\mathrm{F}_{2} \mathrm{O}^{+}$have been studied by near state-of-the-art $a b$ initio calculations. Anharmonic PEFs for the ground state $\left(\tilde{X}^{1} A_{1}\right)$ of $\mathrm{F}_{2} \mathrm{O}$ and the four lowest cationic states have been obtained at the $\operatorname{CCSD}(\mathrm{T}) /$ aug-cc-pVQZ or RCCSD(T)/aug-cc-pVTZ level of theory. Spectral simulations based on both the harmonic and anharmonic oscillator models were carried out to assist the assignment of the observed PE spectrum. The order of the first four cationic states of $\mathrm{F}_{2} \mathrm{O}^{+}$has been firmly established as $\tilde{X}^{2} B_{1},{ }^{2} B_{2},{ }^{2} A_{1}$, and ${ }^{2} A_{2}$, which is the same as that in the valence isoelectronic $\mathrm{Cl}_{2} \mathrm{O}^{+}$.

The IFCA procedure was carried out on the $\mathrm{F}_{2} \mathrm{O}^{+}\left(\tilde{X}^{2} B_{1}\right) \leftarrow \mathrm{F}_{2} \mathrm{O}\left(\tilde{X}^{1} A_{1}\right)$ and $\mathrm{F}_{2} \mathrm{O}^{+}\left({ }^{2} A_{2}\right) \leftarrow \mathrm{F}_{2} \mathrm{O}\left(\tilde{X}^{1} A_{1}\right)$ $\mathrm{PE}$ bands. Experimentally derived geometrical parameters were obtained for the two associated cationic states for the first time. For the $\tilde{X}^{2} B_{1}$ state, the IFCA geometry of $R(\mathrm{FO})=1.323 \pm 0.002 \AA$ and $\angle \mathrm{FOF}=107.2 \pm 0.2^{\circ}$, obtained within the anharmonic model, should be highly reliable. For the ${ }^{2} A_{2}$, state, however, the IFCA geometry $[R(\mathrm{OF})$ $=1.460 \pm 0.50 \AA$ and $\left.\angle(\mathrm{FOF})=95.0 \pm 0.5^{\circ}\right]$ is less reliable, because of the poorly resolved vibrational structure observed in the experimental spectrum. Nevertheless, in view of the wide range of the computed geometrical parameters obtained for the ${ }^{2} A_{2}$ state as discussed (see also Table II), the present IFCA geometry should be currently the most reliable. In this connection, the computed $\operatorname{RCCSD}(\mathrm{T})$ bond lengths for the ${ }^{2} A_{2}$ ionic state are reasonably close to the IFCA value, but the RCCSD $(\mathrm{T})$ bond angles are slightly too large. The computed CASSCF bond angles seem to be superior. 
The second and third PE bands in the He I PE spectrum of $\mathrm{F}_{2} \mathrm{O}$, which correspond to three one-electron ionizations, are heavily overlapping with each other and the vibrational structure is only partially resolved. Nevertheless, based on the simulation, which matches best with the experimental spectrum, the following conclusions can be made. The observed onset of the second band corresponds to the $(0,0,2)$ $\leftarrow(0,0,0)$ position of the ionization leading to the ${ }^{2} B_{2}$ state. Both hot bands and the adiabatic position are too weak to be identified from the observed spectrum. Nevertheless, the AIE position to the ${ }^{2} B_{2}$ state can be deduced from the comparison between the simulated and observed spectra to be $15.71 \mathrm{eV}$. Similarly, the observed VIE component of the second band is mainly due to contribution from the $(1,5,0) \leftarrow(0,0,0)$ and $(2,3,0) \leftarrow(0,0,0)$ vibrational components.

For the third PE band, although the observed AIE and VIE appear to be coincide at $16.44 \mathrm{eV}$ [Fig. 5(a)], spectral simulation suggests otherwise [see Fig. 4(c)]. The contribution from the ionization leading to the ${ }^{2} A_{1}$ state causes the adiabatic component of the ${ }^{2} A_{2}$ ionization to appear stronger than it actually is. The VIE component of the ${ }^{2} A_{2}$ band is the $(1,0,0) \leftarrow(0,0,0)$ vibrational component at $16.54 \mathrm{eV}$. The observed diffuse vibrational features on the low and high IE side of the maximum of the third PE band are very likely due to the overlap with the vibrational structure associated with the ${ }^{2} A_{1} \leftarrow \tilde{X}^{1} A_{1}$ ionization.

From the observed spectrum alone, it is impossible to derive any information on the AIE and/or VIE positions of ionization to the ${ }^{2} A_{1}$ state. Nevertheless, from the highly consistent computed AIEs/ $T_{e}$ values, and the excellent match between the simulated and observed spectra, the AIE and VIE positions to the ${ }^{2} A_{1}$ state should be close to the values of 16.17 and $16.40 \mathrm{eV}$, respectively, as obtained from spectral simulation. Once again, the combination of highlevel $a b$ initio calculations with spectral simulation has been demonstrated as a powerful tool for extracting valuable information from a complex PE spectrum.

\section{ACKNOWLEDGMENTS}

The authors are grateful to the Research Grant Council (RGC) of the Hong Kong Special Administrative Region (Project Nos. POLYU 5156/98P and POLYU 5180/99P) and the Research Committee of the Hong Kong Polytechnic University. One of the authors (D.K.W.M.) would like to thank Professor Kirk Peterson for providing a grid generation program and his help and suggestions in the least square fitting of PEFs. Support from the EPSRC(UK) and the Leverhulme Trust is also acknowledged.

${ }^{1}$ A. B. Cornford, D. C. Frost, F. G. Herring, and C. A. McDowell, J. Chem. Phys. 55, 2820 (1971).

${ }^{2}$ C. R. Brundle, M. B. Robin, N. A. Kuebler, and H. Basch, J. Am. Chem. Soc. 94, 1451 (1972).

${ }^{3}$ K. E. Valenta, K. Vasudevan, and F. Grein, J. Chem. Phys. 72, 2148 (1980).

${ }^{4}$ S. R. Langhoff and D. P. Chong, Chem. Phys. 55, 355 (1981).

${ }^{5}$ D. P. Chong, F. G. Herring, and D. McWilliams, J. Chem. Phys. 61, 78 (1974); 61, 958 (1974).

${ }^{6}$ W. v. Niessen, J. Electron Spectrosc. Relat. Phenom. 17, 197 (1979).
${ }^{7}$ S. R. Langhoff and D. P. Chong, Chem. Phys. Lett. 86, 487 (1982).

${ }^{8}$ P. Duffy and D. P. Chong, Org. Mass Spectrom. 28, 321 (1993).

${ }^{9}$ C.-H. Hu, D. P. Chong, and M. E. Casida, J. Electron Spectrosc. Relat. Phenom. 85, 39 (1997).

${ }^{10}$ D.-C. Wang, E. P. F. Lee, F.-T. Chau, D. K. W. Mok, and J. M. Dyke, J. Phys. Chem. A 104, 4936 (2000).

${ }^{11}$ D. K. W. Mok, E. P. F. Lee, F.-T. Chau, D.-C. Wang, and J. M. Dyke, J. Chem. Phys. 113, 5791 (2000).

${ }^{12}$ F. Motte-Tollet, J. Delwiche, J. Heinesch, M.-J. Hubin-Franskin, J. M. Gingell, N. C. Jones, N. J. Mason, and G. Marston, Chem. Phys. Lett. 284, 452 (1998).

${ }^{13}$ R. Flesch, E. Ruhl, K. Hottmann, and H. Baumgartel, J. Phys. Chem. 97, 837 (1993).

${ }^{14}$ L. Pierce, N. DiCianni, and R. Jackson, J. Chem. Phys. 38, 730 (1963).

${ }^{15}$ Y. Morino and S. Saito, J. Mol. Spectrosc. 19, 435 (1966).

${ }^{16}$ T. J. Lee, J. E. Rice, and C. E. Dateo, Mol. Phys. 89, 1359 (1996).

${ }^{17}$ W. Thiel, G. Scuseria, H. F. Schaefer III, and W. D. Allen, J. Chem. Phys. 89, 4965 (1988).

${ }^{18}$ S. Dressler and W. Thiel, Chem. Phys. Lett. 273, 71 (1997).

${ }^{19}$ J. Breidung, W. Thiel, J. Gauss, and J. F. Stanton, J. Chem. Phys. 110, 3687 (1999)

${ }^{20}$ T. Saarinen, E. Kauppi, and L. Halonen, J. Mol. Spectrosc. 142, 175 (1990).

${ }^{21}$ L. A. Curtiss, K. Raghavachari, G. W. Trucks, and J. A. Pople, J. Chem. Phys. 94, 7221 (1991).

${ }^{22}$ G. A. Petersson, D. K. Malick, W. G. Wilson, J. W. Ocliterski, J. A. Montgomery, Jr., and M. J. Frisch, J. Chem. Phys. 109, 10570 (1998).

${ }^{23}$ M. Kieninger, M. Segovia, and O. N. Ventura, Chem. Phys. Lett. 287, 597 (1998).

${ }^{24}$ D. E. Bernholdt and R. J. Harrison, J. Chem. Phys. 109, 1593 (1998).

${ }^{25}$ L. A. Curtiss, K. Raghavachari, P. C. Redfern, V. Rassolov, and J. A. Pople, J. Chem. Phys. 109, 7764 (1998).

${ }^{26}$ F. Abu-Awwad and P. Politzer, J. Comput. Chem. 21, 227 (2000).

${ }^{27}$ P. Politzer and F. Abu-Awwad, Mol. Phys. 95, 681 (1998).

${ }^{28}$ H. L. Schmider and A. D. Becke, J. Chem. Phys. 108, 9624 (1998).

${ }^{29}$ T. V. Voorhis and G. E. Scuseria, J. Chem. Phys. 109, 400 (1998).

${ }^{30}$ S. A. Kafafi and E.-S. R. H. El-Gharkawy, J. Phys. Chem. 102, 3202 (1998).

${ }^{31}$ S. A. Kafafi, J. Phys. Chem. 102, 10404 (1998).

${ }^{32}$ M. W. Wong, Chem. Phys. Lett. 356, 391 (1996).

${ }^{33}$ M. J. Frisch, G. W. Trucks, H. B. Schlegel et al., GAussian98, Gaussian, Inc., Pittsburgh PA.

${ }^{34}$ P. J. Knowles, C. Hampel, and H.-J. Werner, J. Chem. Phys. 99, 5219 (1993).

${ }^{35}$ MOLPRO is a package of $a b$ initio programs written by H.-J. Werner and P. J. Knowles with contributions from J. Almlöf, R. D. Amos, A. Berning et al.

${ }^{36}$ A. Halkier, T. Helgaker, W. Klopper, P. Jorgensen, and A. G. Csaszar, Chem. Phys. Lett. 310, 385 (1999).

${ }^{37}$ D. G. Truhlar, Chem. Phys. Lett. 294, 45 (1998).

${ }^{38}$ A. Halkier, T. Helgaker, P. Jorgensen, W. Klopper, H. Koch, J. Olsen, and A. K. Wilson, Chem. Phys. Lett. 286, 243 (1998).

${ }^{39}$ D. Feller and K. A. Peterson, J. Chem. Phys. 108, 154 (1998).

${ }^{40}$ H.-J. Werner and P. J. Knowles, J. Chem. Phys. 89, 5803 (1988).

${ }^{41} \mathrm{P}$. Chen, in Unimolecular and Bimolecular Reaction Dynamics, edited by C. Y. Ng (Wiley, New York, 1994), pp. 371-425.

${ }^{42}$ F.-T. Chau, J. M. Dyke, E. P. F. Lee, and D.-C. Wang, J. Electron Spectrosc. Relat. Phenom. 97, 33 (1998).

${ }^{43}$ S. Carter and N. C. Handy, J. Chem. Phys. 87, 4294 (1987).

${ }^{44}$ J. E. Dennis, Jr., D. M. Gay, and R. E. Welsh, ACM Transactions on Mathematical Software, 7 p. 348 (1981); ibid., p. 369.

${ }^{45}$ J. K. G. Watson, Mol. Phys. 15, 479 (1968).

${ }^{46}$ J. K. G. Watson, Mol. Phys. 19, 465 (1970).

${ }^{47}$ F.-T. Chau, D.-C. Wang, E. P. F. Lee, J. M. Dyke, and D. K. W. Mok, J. Phys. Chem. A 103, 4925 (1999).

${ }^{48}$ E. P. F. Lee, D.-C. Wang, and F.-T. Chau, J. Phys. Chem. 100, 19795 (1996).

${ }^{49}$ O. Ruff and W. Menzel, Z. Anorg. Allg. Chem. 190, 257 (1930).

${ }^{50}$ P. Lebeau and A. Damiens, Compt. Rend. Hebd. Seances Acad. Sci. 188, 1253 (1938). 
${ }^{51}$ J. M. Dyke, N. Jonathan, and A. Morris, Int. Rev. Phys. Chem. 2, 3 (1982).

${ }^{52}$ D. K. W. Mok, E. P. F. Lee, F.-T. Chau, and J. M. Dyke, J. Comput. Chem. (submitted).
${ }^{53}$ G. Taubmann, H. Jones, and H. D. Rudolph, J. Mol. Spectrosc. 120, 90 (1986).

${ }^{54}$ H. Burger and G. Schippel, J. Mol. Spectrosc. 121, 238 (1987).

${ }^{55}$ G. Taubmann, Z. Naturforsch. A 42A, 87 (1987). 